\title{
Service für den Einstieg in den fremden Mark† Praktikum bei der deutschen Handelskammer für Spanien
}

\author{
Anne Kathrin Gönner \\ Technical University of Applied Sciences, Wildau
}

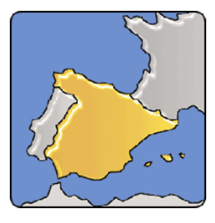

Abstract. Die Autorin berichtet über ihr dreimonatiges Praktikum im Bereich Markt- und Absatzberatung bei der deutschen Handelskammer für Spanien, einem Standort des Netzwerks der Auslandshandelskammern (AHK). Als bilaterale Kammer wird sie als Einrichtung der wirtschaftlichen Selbstverwaltung von Mitgliedsunternehmen in Spanien und Deutschland getragen. Sie fungiert als Vertretung der deutschen Wirtschaft und wirkt in die Wirtschaftspolitik hinein, betreut und koordiniert Kontakte, berät Unternehmen am Markt und beim Export und übernimmt verschiedene Dienstleistungen. Ihr Ziel sind Langzeitbeziehungen zwischen den deutschen und spanischen Unternehmen.

Schlagworte: Auslandshandelskammer, Außenhandel, Deutschland, Dienstleistungen, Interkulturelles Management, Internationales Management, Marketing, Marktanalyse, Praktikum, Spanien, Unternehmensberatung, Verband, Wirtschaftsförderung

Autorin: Anne Kathrin Gönner ist B.A.-Kandidatin im Studiengang Europäisches Management an der Technischen Hochschule Wildau. Sie hat ein deutsch-spanisches Abitur und ein Semester an der Universidad Pontificia Comillas Madrid absolviert.

\section{Einführung}

Praktika bei der deutschen Auslandshandelskammer (AHK) für Spanien sind im Regelfall auf eine Dauer von drei bis acht Monaten ausgelegt und auf die Tätigkeit in einer Abteilung beschränkt. In meinem Fall handelte es sich um eine Stelle im Bereich Markt-und Absatzberatung. Diese muss dauerhaft besetzt sein, da die Anzahl der festangestellten Mitarbeiter nicht ausreicht, um die eingehenden Anfragen zu bearbeiten.

Neben administrativen Tätigkeiten wie Arbeiten an der Rezeption und Beantwortung eingehender Telefonate, gehören vor allem Aufgaben im Bereich Marktforschung, Marktanalysen und Kundenberatung $\mathrm{zu}$ den Schwerpunkten des Praktikums und ergänzen die Arbeit der festen Mitarbeiter.

Hierfür werden insbesondere das Wissen aus dem Bereich Marketing und ausgeprägte Kenntnisse über die politische und wirtschaftliche Situation der beiden Staaten benötigt. Marketingkenntnisse bilden im Wesentlichen die Grundlage für Sonderaufgaben wie Marktanalysen und die Beratung der Unternehmen bezüglich der für sie interessanten Geschäftspartner, da dieses ein Verständnis von Zielgruppen, Konkurrenz, Alleinstellungsmerkmalen und Vertriebswegen voraussetzt. Obwohl all die genannten Themen aufgrund ihrer Komplexität Inhalt verschiedener wirtschaftlicher Fachrichtungen sind, waren während des Praktikums schwerpunktmäßig ihre Marketingaspekte relevant.

Mit dem Ziel, die Auftraggeber umfassend und vollständig beraten zu können, war neben der Erlangung der Informationen auch deren Veranschaulichung erforderlich. Hierbei griff ich insbesondere auf die praxisbezogenen Lehrveranstaltungen (z.B. IT-Anwendungen und Statistik) zurück. Das politische und wirtschaftliche Wissen ist ebenso wenig eindeutig den Fachrichtungen zuzuordnen, da sowohl Themenkomplexe wie allgemeine Betriebswirtschaftslehre als auch Volkswirtschaftslehre und Politik für die tägliche Arbeit vorausgesetzt wurden.

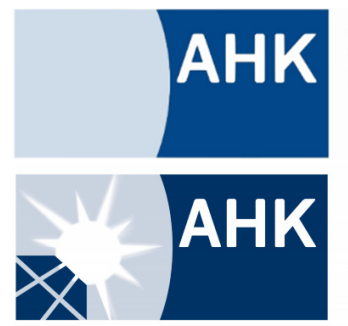

Deutsche

Auslandshandelskammern

Deutsche Handelskammer

für Spanien

Cámara de Comercio Alemana

para España

Für ein Auslandspraktikum typisch, bestimmten neben fachlichen Herausforderungen auch kulturelle Unterschiede und Sprachbarrieren den Berufsalltag des dreimonatigen Auslandsaufenthaltes. Sich die kulturellen Unterschiede zu vergegenwärtigen und mittels Kommunikationsmodellen zu durchdenken war sowohl für das tägliche Miteinander als auch für die Beratung der Kunden notwendig. Ein derartiges Analysieren der Kulturen war essenziell, um Kunden auf die unterschiedlichen Denkweisen der Akteure des fremden Marktes vorzubereiten. Das Thema Pünktlichkeit ist hierbei nur eines, wenn auch das bekannteste, Beispiel für Konfliktpotenzial zwischen Spaniern und Deutschen.

Im Folgenden werde ich erläutern, welche Aufgaben die deutsche Auslandshandelskammer für Spanien hat und in wieweit Praktikanten meiner Abteilung in diese Prozesse involviert werden. Zudem werde ich erläutern, welche theoretischen Grundlagen für die Erfüllung der Aufgaben notwendig sind und welchen Mehrwert ein Praktikum in einem dienstleistungsorientierten Unternehmen mit politischer Relevanz für den Studiengang Europäisches Management und mich persönlich hat. 


\section{Die AHK für Spanien}

„La cámara de comercio alemana para España”, die deutsche Handelskammer für Spanien, ist Teil eines weltweit agierenden Netzwerkes von Wirtschaftsrepräsentanzen Deutschlands, welches sowohl in Form als auch Extension einzigartig ist. Zusammen mit den Auslandshandelskammern bilden verschiedene Delegiertenbüros und einzelne Repräsentanten das so genannte DE80- Netz, welches als Botschafter der deutschen Wirtschaft fungiert. Dieses umfasst 120 Büros in 80 Ländern weltweit, die allesamt unter dem Dach des Deutschen Industrie- und Handelskammertages (DIHK) in Berlin vereint sind. Der DIHK ist auch Dachverband und politische Interessenvertretung der öffentlichrechtlichen Industrie- und Handelskammern.

Das Ziel des Netzwerkes ist es, wirtschaftliche Beziehungen zwischen Deutschland und dem jeweiligen Gastland zu etablieren oder zu festigen. Dank eines vielseitigen Angebots von Dienstleistungen ermöglichen sie deutschen Unternehmen den Einstieg in den Markt des Gastlandes, haben sich jedoch besonders in den letzten Jahren auch vermehrt bemüht, als Ansprechpartner für ausländische Firmen wahrgenommen zu werden. Bei dieser Unterstützung handelt es sich hauptsächlich um Beschaffung von Informationen über den deutschen Markt oder sogenannte Einstiegshilfen. $\mathrm{Zu}$ diesen Einstiegshilfen gehören u.a. Unternehmensberatung, Vermittlung von Wirtschaftskontakten, Informationsbeschaffung, Organisation von Geschäftsreise sowie Veranstaltungen und Kongressen.

Obwohl sich ein Großteil der AHKen selbst verwaltet und durch Mitgliederbeiträge und Serviceleistungen finanziert, erhalten sie zudem ein Leistungsentgelt des Bundesministeriums für Wirtschaft und Technologie. Hierdurch wird deutlich, dass die Aufgaben nicht lediglich privatwirtschaftlicher Natur sind, sondern zudem ein öffentliches Interesse an der Tätigkeit der AHKen besteht. Maßgeblich entscheidend für die finanzielle Situation der AHKen sind jedoch die Beiträge der Mitglieder, wodurch zwangsläufig - wie bei klassischen Wirtschaftsverbänden - die Gewinnung und die Betreuung von Mitgliedsunternehmen zu einem der Haupttätigkeitsfelder wird. Insgesamt finanzieren 40.000 Mitglieder die Kammerarbeit weltweit.

Grundsätzlich sind die Aufgaben der Delegiertenbüros, der einzelnen Repräsentanzen und der AHKen identisch, allerdings definieren lediglich die AHKen die Form und das Ziel der Öffentlichkeitsarbeit in den Gastländern. Andere Institutionen fungieren daher hauptsächlich als ausführende Kraft. Bereits durch die höhere Anzahl der Büros in Relation zu der Zahl der Gastländer wird deutlich, dass in einigen Staaten nicht nur eine AHK ansässig ist, sondern in (aus deutscher Sicht wirtschaftlich interessanten) Ländern mehrere Niederlassungen arbeiten. Hierdurch sollen eine schnelle Bearbeitung der Aufträge und eine individuelle Beratung aller Kunden gesichert werden. Dieses ist in Spanien der Fall: Neben dem Hauptsitz der AHK in Madrid gibt es noch eine weitere Delegation in Barcelona, welche einen Teil der Aufgaben übernimmt (Deutsche Handelskammer für Spanien, 2013, Intranet).

\section{Stellung und Funktionen der AHK}

Die AHK Spanien ist Repräsentant von mehr als 1200 Unternehmen, die in Spanien Investitionen in verschiedenem Umfang getätigt haben. Einige von diesen Firmen arbeiten bereits seit hundert Jahren in diesem Markt. Besonders die letzten Jahre waren aufgrund der Wirtschaftskrise für Unternehmen mit erheblichen finanziellen Einbußen verbunden (Deutsche Handelskammer für Spanien, 2013, Intranet). So stieg das spanische Haushaltsdefizit 2012 auf 6,9 Prozent des BIP (Jones, 2013, S.1), und die Arbeitslosenquote auf rund 27 Prozent. Die Konsequenz waren ein verringerter Import und das Bestreben, den Export zu maximieren (Statista 2013). Nach Spanien exportierende Unternehmen verzeichneten starke Gewinnrückgänge.

Dennoch sind die Nettoinvestitionen deutscher Unternehmen in Spanien laut Pablo García-Berdoy, dem spanischen Botschafter in Deutschland, seit 2010 kontinuierlich gestiegen, da der spanische Markt auch während der Krise für deutsche Investoren interessant erschien. Zudem erhoffte man sich, die Gewinnverluste zu kompensieren, indem man selbst als spanisches Tochterunternehmen auftrat und somit insbesondere spanische Kunden an sich zu binden (García-Berdoy, 2013).

2011 investierten deutsche Unternehmen insgesamt 29 Milliarden Euro in Spanien; aus diesen entstanden Hunderte Millionen Euro an Erneuerungsinvestitionen, welche wiederum langfristige Verbindungen und Abhängigkeiten der beiden Wirtschaften schufen (Plettenberg, 2013, S. 50). Während solcher Investitionsvorhaben oder in wirtschaftlich kritischen Situationen kontaktieren viele mittlere und kleine Unternehmen die AHK Spanien, um von der Expertise und Markterfahrung der Mitarbeiter zu profitieren.
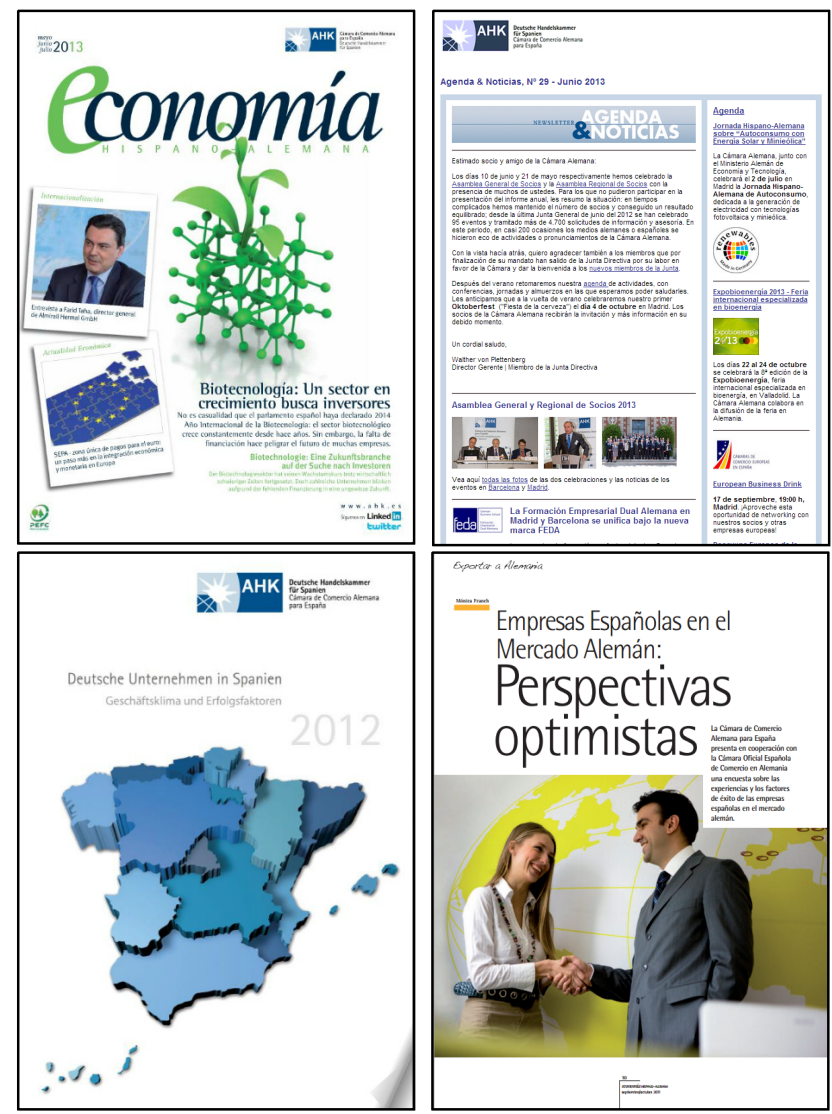

Abbildung 1: Beispiele für Medien der Kammer: Zeitschrift „Economía Hispano-Alemana “ (alle drei Monate), OnlineNewsletter ,Agenda \& Noticias “ (monatlich), Umfragen „,Deutsche Unternehmen in Spanien: Geschäftsklima und Erfolgsfaktoren " und ,Spanische Exporteure auf dem deutschen Markt"

Politisch ist die AHK ein wichtiger Fürsprecher für die „Marke España“ (Deutsche Handelskammer für Spanien, 2013, Intranet). Sowohl in den Publikationen hinsichtlich der medialen Berichterstattung weist die AHK Spanien immer wieder auf die wirtschaftlich positiven Entwicklungen hin, wie z. B. eine nahezu ausgeglichene Leistungsbilanz im Jahr 2012 (Deutsche Bundesbank, 2012, S. 22) oder den Anstieg der Arbeitsproduktivität im Zusammenhang mit einem Rückgang der Lohnstückkosten (Eurostat, 2013). 
Durch die sonst gängige negative mediale Berichterstattung ist eine solche Fürsprache von deutscher Seite notwendig geworden, um deutschen Investoren die Angst zu nehmen, in Verhandlungen mit Unternehmern aus dem krisengeplagten Staat zu treten. Nach dem Medienspiegel der Kammer wurde in führenden Medien allein im letzten Jahr 192-mal auf die AHK und deren Tätigkeit hingewiesen (Deutsche Handelskammer für Spanien, 2013, Intranet).

Um die Koordination mit dem deutschen Staat zu optimieren arbeitet die Kammer eng mit der deutschen Botschaft unter Reinhard Silberberg zusammen. Hierbei handelt es sich hauptsächlich um die Organisation von verschiedenen Veranstaltungen, um den Dialog zwischen deutschen und spanischen Unternehmern anzuregen (Deutsche Handelskammer für Spanien, 2013, Intranet). Georg Oster, Geschäftsführer von Germany Trade \& Investment (GTAI) betont immer wieder, wie essenziell für Spanien die wirtschaftlichen Beziehungen zu Deutschland sind. Nur durch langfristig rentable Investitionen kann die Wirtschaft wieder stabilisiert werden, ebenso kann auf diese Art und Weise das Verlustrisiko von deutscher Seite minimiert werden.

Ein besonderer Schwerpunkt der Kammerarbeit ist die Einführung des in Deutschland bereits praktizierten dualen Ausbildungssystems, welches die erlernte Theorie mit der Unternehmenspraxis kombiniert (Deutsche Handelskammer für Spanien, 2013, Intranet). Spaniens Regierung diskutiert seit Langem die Einführung eines vergleichbaren Systems. Einige Unternehmen, häufig Unternehmen mit deutschem Kapital, praktizieren dieses bereits. Sowohl die Bevölkerung als auch die Politik erhoffen sich, dadurch die Jugendarbeitslosigkeit erheblich reduzieren $\mathrm{zu}$ können und die Einstellung junger Menschen aus Unternehmenssicht attraktiver zu machen. Hierfür wurde von der spanischen Regierung bereits im November 2012 der rechtliche Grundstein gelegt (Servicio Público de Empleo Estatal, 2013, S.1).

Die AHK übernimmt in diesem System zurzeit die Rolle der IHKen in Deutschland, die die Unternehmen bei der Ausbildung unterstützen, sie beraten und die Abschlussprüfung abnehmen. In Zusammenarbeit mit der Berufsschule ASET gelang es in Spanien eine vergleichbare Struktur zu schaffen. Gerade in diesem Bereich wird deutlich, wie eng die Zusammenarbeit mit den deutschen IHKen und der deutschen Regierung notwendigerweise ist (Deutsche Handelskammer für Spanien, 2013, Intranet). Denn nicht nur die spanische Wirtschaft bedarf Unterstützung, sondern ebenso die deutsche, die einen immer gravierenderen Fachkräftemangel aufweist. Eine langfristige Zusammenarbeit mit Spanien könnte die Probleme beider Staaten beheben (Röhl, 2013, S. 3).

Ein weiteres Merkmal der AHK Spanien ist, dass ihre Mitglieder mit einer Anzahl von 1101 (Stand 2012) die dritthöchste der AHKen in Europa ist. Daher weist sie im Jahr 2012 eine Eigenfinanzierungsquote von 93 Prozent auf, die ebenfalls über dem europäischen AHK-Durchschnitt liegt. Dies ist der Grund, warum es sich bei der AHK trotz der Wahrnehmung öffentlicher Belange nicht um eine öffentlich-rechtliche, sondern vielmehr eine privatrechtliche Institution handelt.

Einige der bekanntesten Mitglieder der AHK Spanien sind Robert Bosch España S.A., TÜV Rheinland und Lufthansa. Generell wird unter den Mitgliedern zwischen fördernden und ,normalen" Mitgliedern unterschieden. Die fördernden sind aufgrund ihrer finanziellen Unterstützung über die verpflichtenden Beiträge hinaus zu „socios promotores“, also Fördermitgliedern geworden (Deutsche Handelskammer für Spanien, 2013, Intranet).

Aufgrund der stetig steigenden Anzahl spanischer Kunden ist es für fast alle Angestellten verpflichtend, sowohl fließend Deutsch als auch Spanisch zu sprechen. Insbesondere die Abteilungen Markt- und Absatzberatung, Recht und Steuern und die Geschäftsführung selbst arbeiten bilingual, da sie im direkten Kontakt zu den Kunden stehen und Anfragen aus beiden Ländern bearbeiten. Ein Mindestsprachniveau von $\mathrm{C} 1$ ist deshalb erforderlich. Von dieser Regelung werden im Grundsatz auch Praktikanten nicht ausgenommen.

Neben dem dualen Ausbildungssystem gehört zu den langfristigen Projekten die Etablierung erneuerbarer Energien, ebenso wie die Verbesserung der Infrastruktur mit Schwerpunkt auf Private Public Partnerships. Dies geschieht in erster Linie durch die Organisation verschiedener Konferenzen und Diskussionen, um den spanischen Unternehmen das Thema näher zu bringen und sie dadurch von den Veränderungen zu überzeugen. Ob diese Unterfangen jedoch erfolgreich sind, kann aufgrund des Projektstandes noch nicht beurteilt werden (Deutsche Handelskammer für Spanien, 2013, Intranet).

\section{Praktische Beispiele der Kammerarbeit 2012/2013}

Neben den oben angeführten Großprojekten besteht die Arbeit der AHK aus der Erfüllung alltäglicher Aufgaben, die im Wesentlichen aus der Bearbeitung von Kundenanfragen bestehen. Von diesen wurden allein im vergangenen Geschäftsjahr 4700 bearbeitet, zudem wurden 465 Beratungen durchgeführt und 95 Veranstaltungen zu verschiedenen Themen und Anlässen organisiert. Bei all den genannten Tätigkeiten steht die Einbeziehung der Mitglieder im Fokus, wobei es im Allgemeinen nicht notwendig ist Mitglied zu sein, um die Dienstleistungen der AHK gegen Zahlung eines Entgeltes nutzen zu können.

Die wichtigsten Beispiele für die Kammerarbeit im vergangen Jahr sind zum einen das Unternehmertreffen mit dem Bundesverband der Deutschen Industrie (BDI), dem DIHK und dem spanischen Wirtschaftsministerium zur Strategieentwicklung und Stärkung wirtschaftlicher Beziehungen im September 2012; zum anderen aber auch die Verleihung des deutsch-spanischen Wirtschaftspreis an den Präsidenten von Telefonica. In beiden Fällen handelt es sich besonders um repräsentative Veranstaltungen mit hochrangigen Personen aus Wirtschaft und Politik. Allerdings gehört auch die Organisation von rein wirtschaftlichen Veranstaltungen wie dem Lieferantenforum zum Programm der AHK. Hierbei treffen spanische Lieferanten auf deutsche potenzielle Auftraggeber, um auf diesem Weg den ersten Kontakt herzustellen.

Neben den Veranstaltungen für ein externes Publikum werden auch regelmäßig Veranstaltungen in kleinem Rahmen organisiert, diese dienen im Wesentlichen zur Sicherung der internen Kommunikation. Ein Beispiel hierfür ist das monatliche Treffen der Abteilungsleiter mit dem Geschäftsführer, um aktuelle Geschehnisse zu besprechen, ebenso wie die Generalversammlung der Kammer an jedem ersten Montag eines Monats, bei der die Weitergabe von Informationen der Delegation in Barcelona, Neuigkeiten aus dem Vorstand und Entscheidungen des DIHK in Berlin im Mittelpunkt stehen. Wie in Spanien üblich, schließen sowohl interne als auch externe Veranstaltungen mit einem gemeinsamen Essen und privatem Smalltalk ab, da die persönliche Beziehung Fundament einer jeden Geschäftsbeziehung ist (Deutsche Handelskammer für Spanien, 2013, Intranet).

\section{Interne Struktur der AHK}

Die AHK ist in Bezug auf die Struktur vergleichbar mit einer Vielzahl von Unternehmen. Insgesamt werden in der AHK Spanien in Madrid ca. 30 Mitarbeiter beschäftigt, hinzu kommen immer wechselnde Praktikanten, deren Anzahl zwischen sechs und 16 variiert. Ein Großteil der Beschäftigten sind im Bereich Steuern und Recht und Markt- und Absatzberatung tätig, da dieser über 70 Prozent des Gewinns erwirtschaftet. Alle strategischen Entscheidungen bezüglich Spaniens werden vom Vorstand der Handelskammer für Spanien getroffen, welcher sich aus 23 Vertretern der Wirtschaft und dem Geschäftsführer der Handels- 
kammer zusammensetzt. Walther von Plettenberg, der derzeitige Geschäftsführer ist einfaches Vorstandsmitglied, während Präsident und Vizepräsidenten Repräsentanten deutscher Unternehmen in Spanien sind. Der geschäftsführende Vorstand besteht zudem aus einen Schatzmeister und einen Schriftführer.

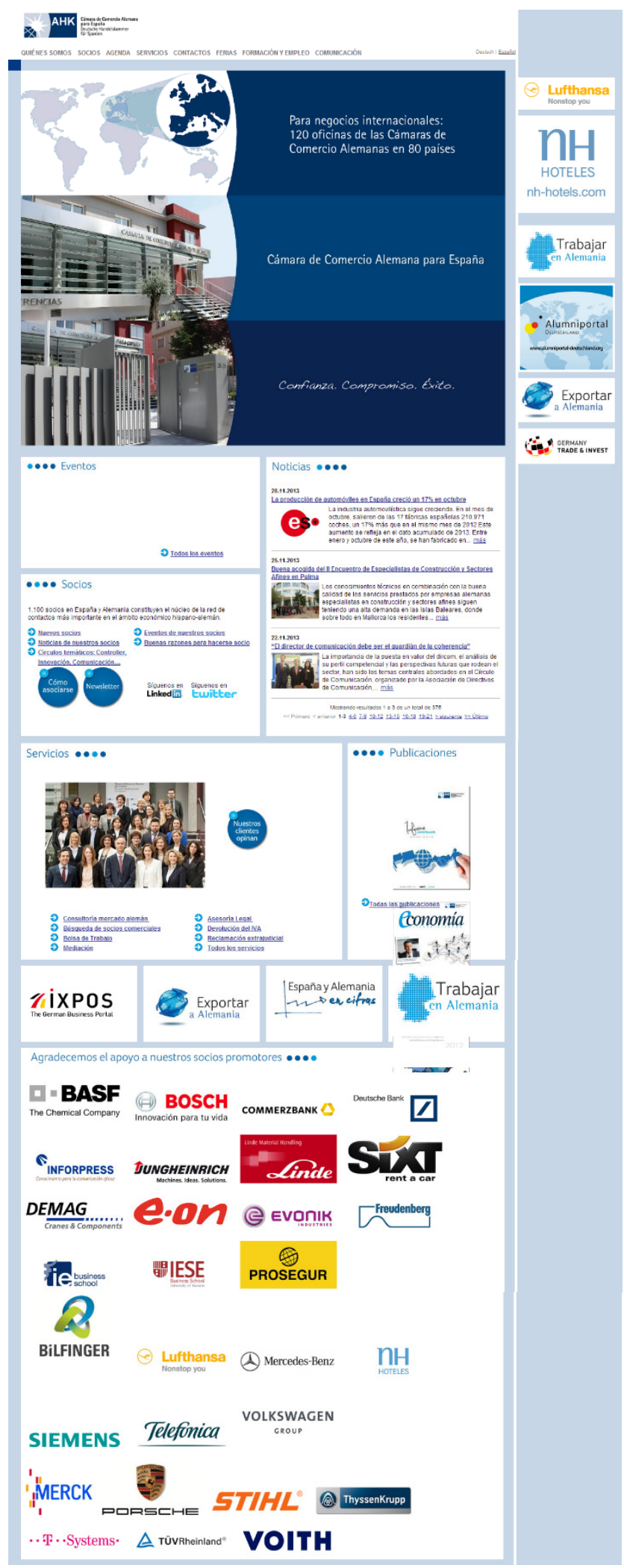

Abbildung 2: Spanischsprachige Website www.ahk.es/es mit aktuellen Nachrichten, Serviceangeboten und Links.
Von der Kammer selbst werden vor allem die mittel- und kurzfristigen Projekte beschlossen, sowie interne Abläufe. Hierbei handelt es sich hauptsächlich um operative Entscheidungen wie das Gestalten von Formularen und des Internetauftritt und der Kammernormen. Hauptverantwortlich für diese Beschlüsse sind neben dem Geschäftsführer seine beiden Vertreter Markus Kemper und Marie Antonia von Schönburg, die zugleich Leiterin der Delegation in Barcelona ist.

Innerhalb jeder Abteilung gibt es zudem eine weitere, wenn auch flachere Hierarchie, die sich theoretisch lediglich durch Abteilungsleiter und Nicht-Abteilungsleiter kennzeichnet. Tatsächlich spielt, wie in spanischen Unternehmen üblich, aber Alter und Geschlecht eine wichtige Rolle bei der Machtverteilung. Obwohl in der Kammer lediglich fünf Männer beschäftigt sind, haben drei von ihnen Führungspositionen und treffen die maßgeblichen Entscheidungen.

Unabhängig von dieser Aufteilung werden zudem generelle Entscheidungen vom DIHK beschlossen und gelten in allen AHKen gleichermaßen - so zum Beispiel im Bereich Kommunikation, Datenspeicherung oder Anfragenbearbeitung. Im Allgemeinen lässt sich also feststellen, dass die AHK durch drei miteinander interagierenden Organe geleitet wird: dem Vorstand, der Geschäftsführung und dem DIHK (Deutsche Handelskammer für Spanien, 2013, Intranet).

\section{Aufgaben im Praktikum}

Die Abteilung Markt- und Absatzberatung unter der Leitung von Markus Kemper besteht aus drei Unterabteilungen: Projektarbeit, Messerepräsentanz und Adresselektion, welche zumeist getrennt voneinander arbeiten und verschiedene Typen von Kunden betreuen. Insgesamt beschäftigt die Abteilung acht Festangestellte, im Sommer 2013 beschäftigte sie zusätzlich drei Praktikantinnen. In allen drei Bereichen sind bis zu drei Angestellte und jeweils mindestens ein Praktikant beschäftigt.

Obwohl die Einheitlichkeit binnen der Abteilungen gewahrt werden soll, ist die inoffizielle Bürosprache bei der Projektarbeit Deutsch, während die Mitarbeiter der räumlich abgetrennten Adresselektion ausschließlich auf Spanisch kommunizieren. Aktueller Schwerpunkt der Abteilung Projektarbeit sind erneuerbare Energien und die Betreuung von Unternehmen des Sektors. Ein attraktiver Aspekt eines solchen Tätigkeitsfeldes sind stetig neue Herausforderungen, durch die ein vergleichsweise abwechslungsreiches Aufgabenspektrum entsteht.

Ähnlich vielfältig ist die Arbeit im Bereich Messerepräsentanz der Messe Stuttgart. Abhängig von der aktuellen Messe wird mit unterschiedlichsten Unternehmen in Kontakt getreten, um diese als Aussteller zu gewinnen. Es handelt sich hierbei aber nicht lediglich um Kundenwerbung für die Messen, sondern vielmehr um eine Rundumbetreuung, die u.a. die Erinnerung an Termine, die Planung des Besuches und die erste Kontaktaufnahme mit potenziell interessanten Geschäftspartnern umfasst.

Der dritte und letzte Bereich Markt- und Absatzberatung war mein Einsatzfeld während des Praktikums. Carlos Aguado, der Leiter des Bereiches Adressrecherche und mein direkter Vorgesetzter, ist in erster Linie verantwortlich für die Bearbeitung alltäglicher Anfragen und den Verkauf von Marktinformationen.

\section{Adressselektion}

Um die Tragweite und die zunehmende Relevanz dieser Unterabteilung zu verstehen, ist es notwendig, den bilateralen Handel der letzten Jahre zwischen Deutschland und Spanien zu analysieren. Innerhalb der letzten zwei Jahre hat der Export deutscher Produkte nach Spanien starken Schwankungen unterlegen. 
Im Jahr 2011 konnte zwar noch eine Steigerung zum Vorjahr von 10,5 Prozent zum Vorjahr verzeichnet werden, jedoch bereits im Folgejahr ein Verlust von 12,9 Prozent. Daraus ergibt sich in absoluten Zahlen ein Importrückgang deutscher Produkte in Spanien im Wert von rund 4.000 Mio. Euro innerhalb eines Jahres. Lediglich ein Warenwert von 27.108 Mio. Euro wird aktuell aus Deutschland importiert, hiermit zählen die deutschen Produkte zwar weiterhin zu den meist importierten Waren Spaniens, gehen jedoch wertmäßig erheblich zurück.

\section{Allgemeine Dienstleistungen der deutschen Handelskammer für Spanien}

Keine der Dienstleistungen wird zum festen Preis angeboten, da trotz gewisser Rahmenvorgaben Preise stets individuell verhandelt werden.

\section{Markt- und Absatzberatung}

Marktanalysen

Geschäftspartnervermittlung

Individuelle Absatzberatung

Exportinitiativen

Terminvereinbarung

Messebeteiligungen

Geschäftspräsenz/virtuelles Spanienbüro

\section{Adressrecherche}

Anzeigenservice

Messeinformationen

Kontaktdaten von Importeuren, Herstellern, Lieferanten

\section{Recht und Steuern}

Basisfragen (kostenfreie Merkblätter)

Individuelle Rechtsfragen

Inkasso

Anwaltsverzeichnis

MwSt-Rückerstattung

Handels-und Registerauskunft

Mediation

Steverliche Betreuung

Personal- und Geschäftsbuchhaltung

\section{Grüner Punkt}

Informationen zur Verpackungsentsorgung

Vermittlung des Abschlusses des nötigen Lizenzvertrages

\section{Bürocenter}

Vermietung von Büroräumen

\section{Personalservice und Ausbildung}

Weiterbildungszertifikate

Jobbörse

Vermittlung spanischer Auszubildender

an deutsche Unternehmen

Personalvermittlung

Hingegen weist die Handelsbilanz einen konstanten Anstieg des Exports spanischer Produkte nach Deutschland auf. Dieses spiegelt sich auch in den relativen Zahlen wider, so weist der exportierte Warenwert ein Steigerung innerhalb der letzten zwei Jahre von über 18 Prozent Steigerung weist der Warenwert auf. Der Exportwert liegt dadurch bereits bei 23.287 Mio. Euro und nähert sich weiter dem deutschen Wert an. Die Verringerung des bilateralen Handelssaldos ist dadurch schrittweise erfolgt (Deutsche Handelskammer für Spanien, 2013, S. 96; Ministerio de
Economía y Competividad, Ministerio de Industria, Energía y Turismo).

Um in ein bisher unbekanntes Land importieren zu können, benötigen die Unternehmen Informationen über den Markt selbst, die Kontaktdaten potenzieller Geschäftspartner und Informationen über die Konkurrenz und deren Marktmacht. Da sich immer mehr Spanier für Deutschland als Wirtschaftsstandort interessieren oder bereits bestehende Beziehungen verändern oder ausweiten möchten, gewinnt die Abteilung Adressrecherche zunehmend an Bedeutung. Sie liefert den Unternehmen die Marktinformationen, welche ihnen diese Vorhaben ermöglichen.

Jedoch sehen auch deutsche Unternehmen in der angeschlagenen spanischen Wirtschaft die Möglichkeit, kostengünstig innerhalb Europas zu produzieren und somit langfristig von der Wirtschaftskrise zu profitieren. Sowohl die Kontaktaufnahme zu den jeweiligen potenziellen Geschäftspartnern beider Staaten als auch die Adressselektion realisierte ich während des Praktikums.

Die Mehrzahl der Kunden sind Mittelständer, die bisher keinerlei oder kaum Erfahrungen auf dem deutschen und zumeist auch nicht auf dem europäischen Markt haben. Sie benötigen somit häufig nicht nur die Dienstleistungen unserer Abteilung, sondern auch die der juristischen Abteilung, um den Export und gegebenenfalls die Errichtung von Zweigstellen organisieren zu können. Ein immer wieder vorkommendes Problem ist jedoch die fehlende Liquidität der Unternehmen. Oft ist es notwendig, die Unternehmer bei der Suche nach Geschäftspartnern dahingehend $\mathrm{zu}$ unterstützen, dass man ihnen im ersten Schritt hilft genau zu definieren, welche Firmen für sie von Interesse sein könnten. Das mangelnde Wissen der Unternehmen lässt nicht zwangsläufig auf fehlende Exporterfahrung schließen, sondern darauf, dass jeder Markt seine Besonderheiten hat. So existieren bestimmte Unternehmen in Deutschland in der Form nicht, in der sie in Spanien üblicherweise vorzufinden sind.

Andersherum gibt es ein beeindruckendes Beispiel: In Spanien existieren faktisch keine Drogerien, da diese in die normalen Supermarktketten komplett integriert sind. Deutsche Unternehmen, die sonst hauptsächlich mit Drogerien zusammenarbeiten, müssen sich dementsprechend in Spanien bezüglich der Vertriebswege und Abnehmer komplett neu orientieren.

\section{Marktanalysen}

Im Folgenden werde ich die Marktanalysen, die ich während meines Praktikums durchgeführt habe an einem konkreten Fallbeispiel erläutern. Solche Analysen waren so genannte Sonderaufgaben, da sie nicht zum alltäglichen Aufgabenbereich zählten und zumeist an andere Abteilungen delegiert wurden. Dennoch war ich während meines Praktikums insgesamt fünf Mal mit vergleichbaren Analysen betraut, die allerdings unterschiedliches Ausmaß hatten.

Der Prozess der Marktanalyse lässt sich in sieben Schritte aufteilen, die sowohl in Bezug auf den zeitlichen Aufwand als auch auf die Komplexität variieren. In diesem Fall hatte sich ein spanischer Uhrenhersteller an uns gewandt, der in näherer Zukunft plante, seine Produkte nach Deutschland exportieren.

\section{Schritt: Informationsbeschaffung über das Produk†}

Um das Segment genau zu bestimmen, war es nötig, telefonisch mit dem Kunden in Kontakt zu treten und genauere Produktinformationen einzuholen. Solche Rücksprachen nach der ersten Kontaktaufnahme sind üblich, da die Unternehmer uns oft lediglich eine grobe Beschreibung ihres Vorhabens und ihrer Produkte zusenden. In diesem Fall sandte uns der Kunde direkt nach dem Telefonat alle benötigten Informationen per E-Mail zu, hierbei handelte es sich sowohl um die technischen Besonderheiten seiner Uhren als auch eine Beschreibung seiner aktuellen Zielgruppe, die Stückzahl und den Preis. Aufgrund des Materials 
war es uns möglich, ein genaues Profil des Kunden und des Produktes zu erstellen.

In diesem Fall war unser Auftraggeber ein Uhrenhersteller, der die Uhrenbänder lediglich aus Leder produziert und ein sehr hochwertiges Uhrenwerk verbaut. Seine sonstigen Produkte ließen sich mit dem Begriff „, Country-Style“ beschreiben. Es war zudem auffallend, dass die Uhren, obwohl sie nach Modeschmuck aussehen, sowohl vom Preis als auch von der Qualität her eher zum Luxussegment zählen. Alle Informationen fasste ich in einer kurzen Präsentation zusammen. Für diesen Abschnitt gibt es innerhalb der Kammer keine genauen Vorschriften, sondern vielmehr überließ mein Chef mir die Beschaffung und Präsentation der Informationen über Produkt und Kunden. Die Präsentation hätte sowohl mit Hilfe von PowerPoint als auch mit einfachen Übersichten aus Word erstellt werden könne, ich entschied mich jedoch aufgrund der Zeitknappheit für letztere $\mathrm{Al}$ ternative.

\section{Schritt: Teambesprechung mit Brainstorming}

Nach der Informationsbeschaffung und Präsentation besprachen wir beim morgendlichen Teammeeting, welche Daten des deutschen Uhrenmarktes für den Kunden interessant sein könnten. Hierbei kamen wir über ein Brainstorming zu dem Entschluss, dass unser Kunde an folgenden Aspekten interessiert sein dürfte: Konkurrenz, Kaufverhalten, Großhändler, Einzelhändler ebenso wie themenspezifische Messen und Zeitschriften.

\section{Schritt: Quellenrecherche}

Im nächsten Schritt musste herausgefunden werden, wie wir diese Informationen beschaffen konnten und welche Quellen in diesem Zusammenhang vertrauenswürdig sind. Als sichere Quellen bezüglich des deutschen Marktes gelten bei der AHK generell alle Informationen des DIHK, der Bundesministerien und im Allgemeinen auch Veröffentlichungen von Dachverbänden des jeweiligen Sektors. Ergänzend wird zudem jedes Mal vorab recherchiert, ob eine andere Institution oder die AHK selbst bereits eine vergleichbare Studie veröffentlicht hatte. Um dies herauszufinden, waren insbesondere eine Internetrecherche und die erste Kontaktaufnahme zu verschiedenen deutschen Uhrenverbänden erforderlich. Diese diente in erster Linie zur Gewinnung einer Übersicht über die beschaffbaren Informationen und denen, die aus datenschutzrechtlichen Gründen nicht oder lediglich gegen ein entsprechendes Entgelt erworben werden können. Daten dieser Art werden aufgrund der Kostenerhöhung von unserer Recherche ausgeschlossen. Des Weiteren stellte ich nach einer Suche in unserem eigenen Archiv fest, dass bisher keine vergleichbare Studie erstellt worden war.

\section{Schritt: Angebot erstellen}

Die Gliederung der Marktanalyse konnte von mir unter Zuhilfenahme meiner Marketingkenntnisse nach den ersten Schritten erstellt werden und wurde dem Kunden umgehend nach der Überprüfung seitens meines Chefs mitgeteilt. Da wir jedoch als privatrechtliche Institution eine entgeltliche Dienstleistung anbieten, war es notwendig, auch ein Angebot zu erstellen. In diesem Fall handelte es sich jedoch um keine einfache Recherche, sondern um eine individualisierte und zeitaufwendige Suche, somit musste der genaue Zeitaufwand vorab kalkuliert werden.

Neben dem Faktor Zeit spielt auch der Faktor Komplexität eine erhebliche Rolle bei solch einer Kalkulation. Die entscheidende Frage, die mir mein Chef bei jedem Punkt der Gliederung stellte, war: „Wie schwer ist es, die Daten zu erhalten und sie entsprechend aufzubereiten, und welchen Zeitrahmen würdest du dafür kalkulieren?" Da mir bekannte Quellen wie Statista, das Bundesamt für Statistik und der Uhrenverband eine sehr beschränkte Anzahl an Informationen boten, kalkulierte ich für die
Zeit pro Gliederungspunkt zwei bis drei Arbeitsstunden á 75 Euro ein. Dies ist der sogenannte Arbeitsdurchschnitt, der dem Kunden die Rechnung und das Verstehen des Angebotes erleichtern sollte.

Jeder Punkt nahm jedoch tatsächlich unterschiedlich viel Zeit in Anspruch. So waren etwa die Konkurrenten mit Hilfe von DIHK-Informationen schnell ermittelt, hingegen waren Angaben bezüglich des Kaufverhaltens sehr widersprüchlich und eine Kontrolle der Daten komplex. Nachdem Zeit und Aufwand angemessen kalkuliert wurden, erstellte ich ein individualisiertes Angebot da es aufgrund der Seltenheit solcher Anfragen keinerlei Vorlagen gab.

\section{Schritt: Rückfragen des Kunden beantworten}

Für den Kunden, obwohl er finanziell sehr gut gestellt war, handelte es sich um einen relativ hohen Kostenpunkt, weshalb er bezüglich der einzelnen Gliederungspunkten erfragte, ob die angebotenen Informationen einen solchen Preis rechtfertigten. Zum Beispiel wollte er wissen, ob mit der Konkurrenz lediglich vergleichbare Hersteller gemeint seien oder jeder deutsche Uhrenhersteller. Alle diese Fragen wurden entweder in Telefonaten oder per E-Mail beantwortet, wobei hierbei zu beobachten war, dass Spanier stets die direkte Art der Kommunikation bevorzugten, da es aus Threr Sicht mehr Vertrauen schafft.

Nachdem der Kunde alle angeforderten Informationen erhalten hatte, erbat er sich Bedenkzeit. Dies ist üblich und bedeutet für die AHK eine Ungewissheit, da bereits viele Arbeitsstunden für die Vorrecherche investiert wurden und bis zu diesem Zeitpunkt unsicher ist, ob überhaupt eine Zahlung des Kunden eingeht. Er entschied sich für die Annahme unseres Angebots.

\section{Schritt: Durchführung der Analyse}

Nach dem Zahlungseingang des Kunden wurde mit der eigentlichen Analyse begonnen, welche durch die ausführliche Vorabrecherche vereinfacht wurde. Die Daten der Konkurrenz, d.h. sowohl die Namen als auch die allgemeine Anzahl und die Verteilung innerhalb Deutschlands wurden per Datenbankrecherche ermittelt. In diesem Fall bot sich die Datenbank des DIHK „FISChrom" an. Sie liefert alle deutschen Unternehmen, die bei einer IHK registriert sind. Im Allgemeinen sind die Daten aktuell und vollständig, d.h. sie beinhalten sowohl eine Beschreibung des Tätigkeitsfeldes der Unternehmen als auch die Kontaktdaten und eventuelle Verfahren wie Insolvenzen. Gesucht wird hierbei mit Hilfe der NACE-Codes, der Statistischen Systematik der Wirtschaftszweige in der EU (Hudetz, Kaapke, 2009, S. 223).

Um an die entsprechenden Codes zu gelangen, werden diese in den von der AHK selbst angefertigten Excel-Listen gesucht und für die Recherche verwendet. Problematisch ist, dass die NACECodes einen kompletten Sektor umfassen, so gibt es in diesem konkreten Fall einen Code für die Herstellung von Uhren, nicht aber etwa einen für die Herstellung von Luxusuhren, die zwar von der Optik her zum Bereich Modeschmuck zählen, aber qualitativ relativ hochwertig sind. FIS bietet dem Verwender jedoch die Möglichkeit, die Suche mittels des Codes um einen Text oder /und eine Beschreibung zu ergänzen. Diese Option ist auf der Programmoberfläche der Datenbank leicht zu finden und entspricht dem Suchbefehl „AND“. Um sicherzugehen, dass die Konkurrenz jedoch komplett erfasst wurde, wurden sowohl die Beschreibung als auch der Text variiert und die Suchergebnisse zusammengefasst. Zudem bietet die Datenbank die Möglichkeit, die Resultate als Excel-Tabelle herunterzuladen, um diese leichter auszuwerten und irrelevante Daten wieder zu löschen.

Allerdings konnte ich auf diese Art und Weise lediglich die in Deutschland ansässige Konkurrenz ermitteln, nicht jedoch ausländische Unternehmen, die ihre Produkte über Großhändler absetzen. Die meisten nahmen jedoch glücklicherweise regelmä- 
Big an Messen des Sektors teil. Deshalb konnte ich durch Anrufe bei verschiedenen Messeveranstaltern in Kombination mit der Liste des FIS eine umfassende Liste der Konkurrenz erstellen. Ebenfalls mit Hilfe des FIS in Kombination mit öffentlich zugänglichen Datenbanken wie „Wer liefert was“, „Wer liefert Wem“ oder den „Gelben Seiten“ war es möglich, die Mehrzahl der deutschen Groß-und Einzelhändler zu erfassen und durch Anrufe bei jedem einzelnen zu erfragen, ob diese theoretisch an dem Typ Uhr des Kunden interessiert wären.

Ein solches Vorgehen war in Bezug auf die Konkurrenz nicht möglich, da diese in keinem Fall über die Exportpläne der spanischen Hersteller informiert sein durfte. Namen des Kunden wurden jedoch aus datenschutzrechtlichen Gründen auch nicht bei den Zwischenhändlern preisgegeben. Durch die Telefonate wurde die Anzahl der Vertriebspartner erheblich reduziert, da die Mehrzahl der deutschen Einzelhändler bereits Exklusivverträge mit bestimmten Fabrikanten hatten oder grundsätzlich nicht an dem Stil des Kunden interessiert waren.

Der nächste Gliederungspunkt bezieht sich auf das Kaufverhalten, welches jedoch in Absprache mit dem Kunden nicht nur das Kaufverhalten des Endkunden beinhalten sollte, sondern ebenso den Aufbau des Marktes und damit verbunden das Verhalten der einzelnen Akteure. Einige Aussagen über das Kaufverhalten ließen sich bereits durch die Telefonate mit den Einzelhändlern treffen, allerdings galten diese lediglich als Meinungen und waren als Quelle nicht belastbar. Hingegen hilfreich waren in diesem Fall Statista und das Bundesamt für Statistik, da auf beiden Plattformen einige Grafiken zum Thema veröffentlicht wurden. So konnte etwa durch die Quellen ermittelt werden, wie viel Geld die Deutschen im Durchschnitt für Uhren ausgeben, woher sie diese beziehen und welche Uhrenhersteller besonders hohe Gewinne erzielen. Letzteres war wichtig, um Rückschlüsse auf den Geschmack der Kunden ziehen zu können.

Allerdings reichten diese Angaben nicht für eine lückenlose Dokumentation, und so bat ich den Dachverband der Uhrenhersteller in Deutschland um Hilfe. Dieser sandte mir eine Verbandsbefragung $\mathrm{zu}$, aus der hervorging, welche Uhrentypen in den letzten zehn Jahren besonders von den Endkunden begehrt wurden. Zudem sandte mir der zuständige Mitarbeiter eine Umfrage $\mathrm{zu}$, aus der hervorging, welche Trends für das kommende Jahr erwartet wurden. Genauere Angaben über den deutschen Markt machte er auch und erklärte mir, wie in den meisten Fällen die Uhren an den Endkunden gelangen. Da es hierfür jedoch keine Dokumente gab, notierte ich mir alle Informationen und überprüfte sie durch stichprobenartige Anrufe bei verschiedenen Händlern.

Im letzten Teil der Marktanalyse suchte ich alle für den Kunden relevanten Messen in Deutschland heraus. Einige von ihnen waren bereits in unseren Datenbanken gelistet und bereits vorab kontaktiert worden, um die Gesamtheit der Konkurrenz zu erfassen. Bei der Suche nach weiteren Messen nutzte ich hauptsächlich Information des Ausstellungs- und Messe-Ausschusses der deutschen Wirtschaft (AUMA). Insbesondere durch spanischsprachige Publikationen konnte ich diese Informationen leicht für unseren Kunden aufbereiten. Durch eine einfache Stichwortsuche erhielt ich sowohl eine Liste von Messen rund um das Thema Uhren als auch eine weitere zum Thema Luxusgüter mit Schwerpunkt Schmuck. Auf diesen Messen waren auch die Herausgeber verschiedener Fachzeitschriften vertreten, an deren Kontaktdaten ich durch einen Anruf bei AUMA gelangen konnte.

\section{Schritt: Datenaufbereitung und Redaktion}

Nachdem ich alle Daten für den Kunden gesammelt hatte, musste ich diese für ihn aufbereiten. Hierbei lag der Schwerpunkt auf Verständlichkeit und Übersichtlichkeit. Laut Vorgabe meines Chefs sollte der Bericht eine Mischung aus Diagrammen, Texten und Tabellen sein. Selbstverständlich mussten hierfür vorab die
Informationen auf Spanisch übersetzt werden. Neben den Adressen, die ja bereits in Tabellenform vorlagen, übertrug ich die Daten in Diagramme und ergänzte diese durch erklärende Texte.

All diese Texte mussten vor Versenden von meinem Vorgesetzten kontrolliert werden, um Verfälschungen durch Übersetzungsfehler zu vermeiden. Im letzten Schritt musste ein ansprechendes Layout entworfen werden, welches einheitlich und passend zum allgemeinen Layout der AHK ist. Als der Bericht vorlag, gab es eine abschließende Teambesprechung, bei der die Zeitangabe überprüft wurde, ebenso wie die Quellenangaben. Leider hatte ich mich bezüglich der Zeit minimal verkalkuliert, was im Wesentlichen an der mangelnden Erfahrung in dem Bereich liegt.

Im Allgemeinen ist eine Marktanalyse eng mit Kommunikation und Recherche verbunden und wird um die individuelle Vorund Aufbereitung der Daten ergänzt. Aufgaben dieser Art sind typisch für die AHK, da die Unternehmen durch die gelieferten Informationen den Markteintritt besser gestalten und zudem das Risiko adäquat berechnen können.

\section{Adressrecherchen und Kontaktaufnahmen}

Neben den Marktanalysen, die den komplexesten Aufgabenbereich meines Praktikums darstellen, gehörten einfache Adressrecherchen und die Erstkontaktaufnahmen mit potenziellen Geschäftspartnern $\mathrm{zu}$ meinen Routineaufgaben. Während die Adressrecherchen oft einen Teil einer kompletten Marktanalyse darstellen, sind die Kontaktaufnahmen mit den Unternehmen durch andere Herausforderungen gekennzeichnet.

Adressrecherchen werden aufgrund zweierlei Anfragetypen angeboten. In dem ersten Fall sucht ein Kunde die Kontaktdaten deutscher Großhändler, Fabrikanten, Einzelhändler und Verbände des Produkts X. Eine solche Suche ist unter der Zuhilfenahme der oben beschriebenen Suchmaschinen und der Informationen der Verbände sehr schnell zu realisieren.

In den meisten Fällen sind die in Auftrag gegebenen Suchen jedoch nicht allgemein gehalten, sondern es handelt sich um ganz spezifische Produkte, über die die von uns verwendeten Datenbanken keinerlei Auskunft geben. So suchten wir zum Beispiel für einen Kunden nicht lediglich die Hersteller von Plastikflaschen, welche unter einem bestimmten NACE-Code registriert werden, sondern Hersteller von Plastikflaschen des Typs X. In einem solchen Fall entsteht für den Kunden ein Aufpreis, da die Recherche mit mehr Zeitaufwand verbunden ist.

Es ist hierbei erfahrungsgemäß notwendig, die Unternehmen selbst zu kontaktieren, um zu erfahren, ob sie ein solches Produkt vertreiben oder herstellen könnten. Hierfür muss die genaue Produktbeschreibung übersetzt werden, Telefonate oder EMailverkehr mit den Firmen geführt werden und schließlich eine Vorabüberprüfung der Konditionen stattfinden.

Um den Kreis der zu kontaktierenden Unternehmen so klein wie möglich zu halten, wird eine reine Adressselektion mittels eines übergeordneten NACE-Codes vorgenommen. Sobald auf unsere E-Mails, Telefonate oder die Kommunikation mit den örtlichen Handelskammern Reaktionen kommen, wird der Kunde nach einer kurzen Überprüfung informiert.

Allerdings werden hierbei ausschließlich Kommunikationsmöglichkeiten und sowohl Kompetenzen als auch Kapazitäten überprüft, hingegen bleiben Preisverhandlungen außen vor, da diese zwischen den Unternehmen stattfinden müssen.

Ein immer wieder auftretendes Problem in diesem Zusammenhang war, dass spanische Unternehmer häufig weder auf Englisch noch auf Deutsch verhandeln können und zudem nicht bereit sind, eine Arbeitskraft einzustellen oder diese Aufgaben an ein Übersetzungsunternehmen zu vergeben. Leider mussten wir feststellen, dass diese dann lieber auf lukrative Aufträge verzichteten! 
Ein konkretes Beispiel für die so genannte „Gestión“ oder auf Deutsch: erweiterte Adressrecherche, war ein deutsches Unternehmen, welches Modellfahrzeuge in Spanien herstellen lassen wollte. Es handelte sich hierbei sowohl um die Herstellung einzelner Teile als auch die Montage. Zudem gab uns das Unternehmen eine weitere Einschränkung vor. Die Modelle sollten ausschließlich aus Zink produziert werden, und zwar reines Zink, womit ähnliche Stoffe wie Zamak ausschieden. Das Unternehmen hätte eine reine Adressrecherche anfordern können, deren Resultat aber lediglich die Kontaktdaten von Herstellern von Metallerzeugnissen geliefert hätte. Selbst wenn die AHK gegen ein erhöhtes Entgelt das Kriterium Zink berücksichtigt hätte, wären mindestens 80 Prozent der Unternehmen aufgrund ihres Profils ungeeignet gewesen.

Wir ließen uns deshalb von dem deutschen Unternehmen, welches im Folgenden Zinkmodell genannt werden wird, einen Teil seines Kataloges und die genaue Beschreibung der Produkte zusenden. Mit Hilfe dieser Materialien erstellten wir einen Brief an potenzielle Geschäftspartner, in dem wir anfragten, ob diese solche Produkte herstellen könnten und an der Kooperation mit einem deutschen Unternehmen interessiert wären. Hierbei mussten die relevanten Daten zuerst auf Spanisch übersetzt werden, was aufgrund der erforderten technischen Kenntnisse nur unter Zuhilfenahme verschiedenster Übersetzungsquellen möglich war. Im Anhang an diesen Brief befanden sich die entsprechenden Bilder des Kataloges, um jede Form von Missverständnissen zu vermeiden. Umso präziser und genauer unsere Anschreiben war, desto relevanter waren die Antworten der Unternehmen.

Nach der Vorbereitung des Anschreibens starteten wir ein Mailing sowohl per E-Mail als auch per Post und kontaktierten zusätzlich alle uns bekannten „Cámaras de comercio“ in Spanien, also das spanische Pendant zu den IHKen, und fragten, ob diese unter ihren Mitgliedern einen Unternehmer hätten, der ein solches Projekt realisieren könnte und wollte. Zusätzlich bietet die Plattform „Subcontratación“ die Möglichkeit, einen Steckbrief hochzuladen der interessierte Unternehmen ansprechen sollte, hierfür wurde das Anschreiben abermals verändert. Der Vorteil dieses Steckbriefes ist, dass die Information vor allem von Unternehmen gefunden wird, die bereits nach gewissen Stichworten suchen und dadurch wahrscheinlich für unseren Kunden von Interesse sein würden.

Die Adressen der angeschriebenen Unternehmen erhielten wir durch die Suchmaschinen, die wir für den spanischen Markt verwendeten, die vom Aufbau vergleichbar mit dem FIS sind. Eine solche ist „FOM“, bei der die 25.000 größten spanischen Unternehmen aus verschiedenen Bereichen registriert sind. Diese arbeitet ebenfalls mit den CNAE (NACE auf Spanisch) und der zusätzlichen Ergänzung eines Textes. Die Resultate sind sehr ausführlich, da sie auch den Umsatz, die Zahl der Angestellten und ähnliche Informationen über die Unternehmen beinhalten. Neben dieser Suchmaschine verwendeten wir „Kompass“, welches eine B2B- Plattform ist. All diese Adressen nutzen wir, um möglichst viele Unternehmen, die im Bereich Schweißen, Montage oder Metallverarbeitung tätig waren, zu kontaktieren. Zusätzlich zu den Mailings führten wir Telefonate durch.

Das Resultat waren die Antworten von sieben Unternehmen bei einem Arbeitsaufwand von fünf Werktagen. Durch die Vorabüberprüfung mussten wir jedoch feststellen, dass zwei Unternehmen leider nicht in der Lage waren in Verhandlungen $\mathrm{zu}$ treten, da sie inzwischen lediglich mit dem billigeren Zamak arbeiten bzw. aufgrund von Sprachbarrieren keine Verhandlungen mit der Firma Zinkmodell führen konnten. Die Daten der restlichen Unternehmen wurden in Tabellenform gespeichert und der Firma Zinkmodell übermittelt, welchen wir zudem in einem Abschlusstelefonat die Resultate und die Reaktionen der Unternehmen mitteilten.
Als zusätzlichen Service wiesen wir zudem auf die Problematik des spanischen Marktes hin - wie mangelhaftes Englisch oder eine ausgeprägte Spontanität. Um die Zufriedenheit des Kunden zu gewährleisten, riefen wir zwei Wochen später abermals an, da wir erfragen wollten, ob die Verhandlungen Früchte getragen hatten, was bei diesem Unternehmen der Fall war.

Eine solche Suche hat sowohl einen entscheidenden Vorteil, als auch einen erheblichen Nachteil gegenüber anderen Suchformen. Der Vorteil ist die Eignung der Suchresultate, während der Nachteil die geringe Anzahl an Resultaten ist. Im Allgemeinen wird eine solche Suche von der AHK empfohlen, da sie dem Unternehmen viel Zeit erspart und die späteren Verhandlungen erleichtert. Im Verhältnis dazu ist der Aufpreis gering, da eine allgemeine Suche, je nach Komplexität bis zu 300 Euro kostet und die AHK eine „Gestión“ im Allgemeinen zu einem Preis von 600-1000 Euro anbietet. Diese Form der Suche beinhaltet neben der Recherche noch den direkten Kontakt zu potenziellen Geschäftspartnern und erspart dadurch den Kunden die Arbeit, die sie mit Suchergebnissen einer standardisierten Suche hätten.

\section{Weitere Kundenbetreuung}

Jede der Dienstleistungen der AHK wird ergänzt durch eine intensive Kundenbetreuung. $\mathrm{Zu}$ dieser gehören sowohl die regelmäßige Information über den Stand unserer Arbeit als auch die Nach- bzw. Rückfragen bezüglich der Angaben der Unternehmen. Letzteres dient in erster Linie dazu, dem Kunden die tatsächlich interessanten Marktinformationen liefern zu können und ihn mit weiteren, bisher von ihm nicht angeforderten Informationen über den ihm noch fremden Markt zu beliefern.

$\mathrm{Zu}$ der bilingualen Kommunikation während des Praktikums gehörten neben der Betreuung der Kunden im Bereich Marktund Absatzberatung auch die mit dem administrativen Aufgabenfeld verbundenen Konversationen. Beispiele hierfür sind der Empfang von Besuchern oder das Beantworten verschiedener externer Anrufe, bei welchen ich regelmäßig mit überwiegend spanischsprachigen Personen kommunizierte. Unter den Besuchern waren sowohl Kunden der AHK als auch Vertreter der deutschen Botschaft oder des Ministeriums für Wirtschaft und Technologie.

Ähnlich vielschichtig waren die Telefonate, bei denen hauptsächlich allgemeine Informationen über die Auslandshandelskammer, die verschiedenen Prozesse, Kosten und Tätigkeitsfelder erbeten wurden. Im Laufe des Praktikums war es möglich, immer mehr selbstständig zu beraten und Anfragen lediglich bei sehr spezifischen Themen an Kollegen weitergeben zu müssen.

\section{Aus dem Studium ins Praktikum: Wie viel Theorie ist anwendbar?}

Um die beschriebenen Aufgaben bestmöglich erfüllen zu können, war es nötig auf bereits erlerntes theoretisches Wissen zurückzugreifen. Zur genaueren Analyse der theoretischen Aspekte, die in die Lösung mit eingeflossen sind, werden die Aufgaben drei übergeordneten Kategorien zugeteilt und im Anschluss auf ihre jeweilige zugrunde gelegte theoretische Basis untersucht.

Diese drei Gruppen sind: Marktanalysen, die Recherche sowie Kommunikation und Beratung. Obwohl eine Marktanalyse im Allgemeinen auch die beiden anderen Themen beinhaltet, so stehen nicht diese, sondern inhaltliche Schwerpunkt bei der folgenden Begutachtung im Fokus. 


\section{Marktanalyse}

Bei der Marktanalyse geht es im Wesentlichen darum, dem Kunden einen Überblick über den Teil des Marktes zu verschaffen, der für ihn und seine Unternehmung relevant ist. Da meine Unterabteilung vorher kaum solche Aufgaben selbst bearbeitet hatte, durfte ich das Konzept für eine solche Analyse selbst entwickeln. Hierfür bediente ich mich meines Marketingwissens aus dem dritten Semester. Aufgrund der allgemeinen Gültigkeit des Grundwissens, welches wir im Marketingkurs erlernt hatten, war es möglich, einzelne Punkte des Unterrichtsplanes auf unseren konkreten Fall zu übertragen.

Generell nutzte ich daher zur Erarbeitung der Gliederung die Theorie des Marketing-Mix in Kombination mit der Definition des Begriffs Markt und den Einflussfaktoren auf ein Unternehmen. All diese behandelten Aspekte haben einen direkten oder indirekten Einfluss auf das Handeln eines Unternehmens.

Da unser Kunde das erste Mal auf dem deutschen Markt auftreten wollte, musste er also all diese Aspekte des Marktes für seine Planung berücksichtigen. Relevant waren hierbei unter anderem die sieben Komponenten des Marketing-Mix, deren Zusammenwirken maßgeblich entscheidend für den Erfolg des Produktes ist. Diese Faktoren werden zumeist auch als ,sieben Ps“ bezeichnet: Product, Price, Promotion, Place, People, Process, Physical Facilities - in der deutschen Variante: Produkt, Preis, Promotion, Vertrieb, Personal, Prozessmanagement und äußeres Erscheinungsbild (Meffert; Burmann; Kirchgeorg, 2012, S. 22).

Da das Produkt in unserem Fallbeispiel bereits vorgegeben war, bleibt hierbei lediglich die Information und Analyse der Produkte, die bereits auf dem deutschen Markt vorzufinden sind. Es handelt sich hierbei also im Wesentlichen um eine Analyse der Konkurrenz: Welche Produkte werden in welcher Stückzahl auf dem deutschen Markt von ähnlichen Wettbewerbern bereits abgesetzt (Kuß; Tomczak,; Reinecke, 2008, S. 222)? Der Aspekt Produkt konnte also nicht direkt auf eine Marktanalyse angewandt werden, da die AHK bis auf Modifizierungsvorschläge keinerlei Beratungsfunktion bezüglich der Ware selbst wahrnimmt.

Hingegen ist für eine derartige Analyse der Preis relevant. Zwar wurde auch hier von Seiten des Kunden ein Rahmen vorgegeben, welcher durch die hohe Qualität des Materials auch berechtigt erschien, aber dieser konnte im Gegensatz zum Produkt an den neuen Markt angepasst werden. Um diese Anpassung erfolgreich vorzunehmen, benötigt der Unternehmer die Information, zu welchen Preisen vergleichbare Produkte auf dem fremden Markt angeboten werden und wie viel Geld die potenziellen Kunden bereit sind, in ein solches zu investieren. Im Allgemeinen beinhaltet dieser Teil des Marketing-Mix ebenfalls alle Zahlungswege, Informationen über Rabatte und regionale Preisunterschiede (Söllner; Wilfert, 2001, S. 470-488).

Auch diese Aspekte berücksichtigte ich in meiner Marktanalyse, indem ich marktübliche Konditionen für Groß-und Einzelhändler sowie Gewinnmarge und in Deutschland übliche Zahlungsmittel ergänzte. Der preisliche Aspekt und alle damit verbunden Daten werden besonders ausführlich behandelt, damit eine angemessene Kalkulation der Exportkosten möglich ist.

Ebenso entscheidend wie der Preis ist die Distribution. Zum Vertriebsweg gehören alle Zwischenstationen zwischen dem Fabrikanten und dem Endkunden. Für Unternehmer ist im Wesentlichen relevant, welche Arten von Distributionswegen es gibt und wie weit diese genutzt werden. Zu Distribution gehören im Besonderen die Verkaufsstellen, in denen der Endkunde das Produkt erwirbt, und die Großhändler (Pufahl, 2006, S. 136).

Hierbei reicht es nicht, dem Kunden lediglich Kontaktdaten, sondern viel mehr kurze Informationen über die einzelnen Stationen zu liefern. Dieses ist besonders für ausländische Investoren von Bedeutung, da ihnen die ausschließlich in Deutschland agierenden Geschäfte nicht bekannt sind.

Der vierte Aspekt ist die Promotion, die weit mehr beinhaltet als reine Produktwerbung. Vielmehr umfasst dieser Begriff alle Bereiche, die für die Produktvermarktung notwendig sind. Zum einen bedarf es daher Informationen über die Medien und Wege, mittels derer das Produkt beworben wird und mit welchen Kosten ein solcher Prozess verbunden ist, zum anderen umfasst der Begriff aber auch die Markenführung, also das Branding (Hiller; 2007, S. 21)

Um möglichst effektiv zu werben, ist es notwendig zu analysieren, an welchen Arten von Marken der deutsche Einkäufer interessiert ist und inwiefern man die Marke in welcher Form unabhängig vom Produkt selbst in Deutschland verkaufen sollte. Die Herausforderung ist es hierbei, die bereits existierende Marke an den neuen Markt anzupassen, ohne komplett ihren Erkennungswert aufzugeben (Tropp, 2011, S. 59).

$\mathrm{Da}$ ich mich bei meiner Schwerpunktsetzung für den erweiterten Marketing-Mix entschieden habe, beinhaltet meine Analyse ebenfalls die Punkte äußeres Erscheinungsbild, Personal und Prozessmanagement. Diese sind hauptsächlich für Unternehmer relevant, die selbst Zweigstellen eröffnen möchte, weil es sich hierbei im Allgemeinen um die Ausgestaltung des Ladenbetriebes handelt. Das äußere Erscheinungsbild bezieht sich auf den Aufbau und das Design des Ladens, welche stets an die Zielgruppe angepasst werden sollten. Sowohl Dekoration als auch Raumgestaltung und Atmosphäre sind hierbei zu beachten. Eng hiermit verbunden ist der zweite Faktor Personal, da dieses die Kaufentscheidung des Kunden ebenso wie die Nachkauferfahrung maßgeblich beeinflusst. Wie qualifiziert muss das Personal sein, wie viel Berater müssen angestellt sein um die Fragen der Kunden beantworten und in welcher Art und Weise sollten sie auf Beschwerden reagieren? Für diesen letzten Punkt ist das Verständnis für die andere Kultur entscheidend (Simon, 2009, S. 146-151).

Der dritte und letzte Aspekt, das Prozessmanagement, umfasst sämtliche Vorgänge, die mit dem Kauf oder der Rückgabe des Produktes verbunden sind. Neben gesetzlichen Regelungen bestimmen auch die örtlich üblichen Verfahren diesen Punkt. Diese beiden Aspekte müssen dem Kunden anhand der Analyse näher gebracht werden. Ein serviceorientierter Prozess überzeugt Kunden abermals in dem Geschäft einzukaufen und schafft somit eine Loyalität (Senden; Dworschak; 2012, S. 47-61).

Abgesehen von den Kategorien des Marketing-Mix beeinflussen weitere externe Faktoren das Handeln eines Unternehmens. Solche sind zumeist eng mit dem Aufbau und Wirken des Marktes selbst verbunden. Mittels dieser Faktoren ist es also weitestgehend möglich den Markt zu definieren.

Es handelt sich hierbei um die demographischen Faktoren, gesetzlichen Regelungen, politische Situation, soziokulturelle Gegebenheiten ebenso wie um wirtschaftliche Struktur und die Ressourcen des Staates.

Während man sich bei der politischen und wirtschaftlichen Situation auf allgemeine Daten berufen kann, welche für die AHK sehr leicht zu beschaffen sind, benötigt die Analyse der weiteren Punkte eine spezifische Bewertung aus Sicht des Kunden. Sie dient im Wesentlichen zur Bestimmung der Zielgruppe und der Planung der Prozesse. Für Letzteres sind insbesondere die gesetzlichen Regelungen und das Vorkommen verschiedener Ressourcen relevant.

Unter gesetzliche Regelungen fallen zum Beispiel alle Richtlinien und Gesetze zum Verbraucherschutz ebenso wie Steuern und Subventionen. Diese Aspekte überschneiden sich in Teilen mit denen des Marketing-Mix, stellen dadurch jedoch eine Ergänzung der Gliederung dar (Freiling, Reckenfelderbäumer, 2007, S. 199-202). 
Vergleichbar in ihrer Funktion ist die Segmentierung, die im Zusammenhang mit den, aus den vorherigen Schritten gewonnenen, Erkenntnissen eine Zielgruppe definiert. Berücksichtigt hierbei wird die demographische Zusammensetzung der Käufer (Alter, Herkunft, etc.), deren soziale und wirtschaftliche Situation ebenso wie ihr Kaufverhalten und ihre Persönlichkeit (Griese; Bröring, 2011, S. 71-83). Eine solche Studie benötigt viel Zeitaufwand, darum werden in diesem Fall entweder Dritte mit der Analyse beauftragt oder auf die bisherigen Ergebnisse anderer Institutionen zurückgegriffen.

Insgesamt lassen sich aus der Kombination dieser Kriterien und dem Bewusstsein über deren Wirkung sowohl der allgemeine Sinn als auch die Gliederung und Funktion einer Marktanalyse ableiten.

\section{Recherche}

Die Recherche ist unabdinglich für jede Marktanalyse, da sie die Voraussetzung ist, um an die im ersten Schritt über die Marketingtheorien bestimmten Marktdaten zu gelangen. Während die Gliederung basierend auf den oben beschriebenen Theorien erstellt wird, ist Recherchearbeit praxisbezogen und verändert sich abhängig von den zur Verfügung stehenden Datenbanken, Suchmaschinen oder Anlaufstellen. Dennoch nutzte ich mehrheitlich IT-basierte Mittel zur Informationsbeschaffung.

Um möglichst schnell und effektiv die gewünschten Ergebnisse zu erhalten, ist ein technisches Grundverständnis erforderlich, welches wir uns im Studium in zwei IT-Kursen angeeignet haben. $\mathrm{Zu}$ diesem Grundverständnis gehören unter anderem die Funktionsweise von Suchmaschinen, die Nutzung von Datenbanken und die Vorteile eines klar strukturierten Wissensmanagements.

Da Internet-Suchmaschinen generell nicht das komplette Netz erfassen, sondern Crawler lediglich einen bestimmten Bereich durchsuchen, ist es wichtig, so viele Suchmaschinen wie möglich $\mathrm{zu}$ nutzen, um vielfältige und vollständige Informationen $\mathrm{zu}$ erhalten. Ebenso muss beachtet werden, dass die Auflistung nicht auf der Relevanz der Ergebnisse basiert, sondern käuflich erworben werden kann oder lediglich die Häufigkeit der Klicks widerspiegelt. Diese Funktionsweise führt dazu, dass aktuelle Informationen zumeist nicht auf den ersten Listenplätzen erscheinen (Müller; Plieninger; Rapp, 2013, S. 22). Aufgrund dieser Tatsache war es hilfreich, Alternativen wie Verzeichnisdienste wie z. B. Yahoo zu nutzen, da diese strukturierter aufgebaut sind. Im Wesentlichen arbeiten sie mit einem Oberbegriff, welcher zu weiteren Unterverzeichnissen führt (Kemper; Baars; Mehanna, 2010, S. 154).

Ein solcher Aufbau ist für die Recherche innerhalb eines bestimmten Sektors sehr effektiv, da er zu untergeordneten Aspekten führt und somit einen Gesamteindruck über den Sektor bietet. Nach Abwägung der Vor- und Nachteile war eindeutig, dass kommerzielle Datenbanken wie z.B. Kompass oder „Wer zu Wem" die wertvollsten Ergebnisse liefern und lediglich für den Fall, dass eine Suchanfrage zu spezifisch ist, andere Quellen wie z.B. das Internet als Hauptquelle genutzt werden. Dennoch kam es während meines Praktikums wiederholt vor, dass auf „unsicherere Quellen“ zurückgegriffen wurde. Eine Suche in den gängigen Suchmaschinen und Verzeichnissen wird in diesen Fällen durch die Boole'schen Operatoren vereinfacht und führt zu bestmöglichen Ergebnissen. Dies sind feste Abfrageoptionen, welche genau definieren was im Ergebnisdokument vorkommt. Hierdurch werden sowohl die Ergebnisse limitiert als auch relevanter. Der Informationsgehalt der gefundenen Dokumente ist dementsprechend höher. $\mathrm{Zu}$ den besagten Operatoren gehören Suchbefehle wie AND, OR, NOT.

AND bedeutet, dass die Ergebnisse beide Begriffe beinhalten müssen, während NOT heißt, dass der erste Begriff vorkommen soll, während der zweite nicht vorkommen darf. OR umfasst deutlich mehr, da sowohl der erste Begriff als auch der zweite vorkommen dürfen. Ebenso nützlich ist die sogenannte Phrasensuche mittels Anführungszeichen, da eine solche Suche lediglich Ergebnisse liefert, in denen die komplette Phrase vorkommt (Müller; Plieninger; Rapp, 2013, S. 17).

Eine solche Vorgehensweise wurde in der beschriebenen Marktanalyse im Rahmen der Internetrecherche angewandt. Aufgrund dieser Methoden war es möglich, die Ergebnisse zu begrenzen. Suchbegriffe wie beispielsweise "Uhr AND Leder“ führten zu Webseiten, die für die Analyse wertvoll waren. In diesem Zusammenhang ist es wichtig, dass vorab genau analysiert wird, welches Segment und welche Informationen benötigt werden. Die praktische Hilfestellung aus dem IT-Kurs ermöglichte in einem darauffolgenden Schritt die effiziente Suche. In diesem Zusammenhang wird deutlich, wie eng die Analyse mit der Recherche und ebenfalls den scheinbar vollkommen unabhängigen Wirtschaftstheorien verbunden sind.

Ebenfalls erforderlich war eine ansprechende und verständliche Aufbereitung der Rechercheergebnisse, welches mittels Excel-Tabellen realisiert wird. Funktionen wie das Erstellen von Diagrammen oder das programmierte Ordnen von Daten machen es möglich, den Zeitaufwand für den Abschnitt so gering wie möglich zu halten. Einen Großteil der erforderlichen Kenntnisse erwarb ich bereits in den Mathematik-Vorlesungen. Im Praktikum konnte ich dieses Wissen durch ständiges Anwenden festigen.

\section{Interkulturelle Kommunikation und Beratung}

Im Bereich der Kommunikation ist unabhängig von den erforderlichen Sprachkenntnissen ein kulturelles Verständnis von Vorteil. Hierfür ist es essenziell zu verstehen, warum Personen aufgrund ihres kulturellen Hintergrundes anders agieren als Personen des fremden Kulturkreises. Um Kunden aus beiden Kulturkreisen adäquat beraten zu können, war es notwendig, die Lebensformen in beiden Länder zu studieren und Unterschiede herauszuarbeiten.

Ich nutzte zwei Theorien aus der Lehrveranstaltung „International Communication and Presentation“. Das Black-Box-Modell (Schulz von Thun) basiert u.a. auf der Annahme, dass ein Jeder eine eigene „Black Box“ besitzt, welche in Abhängigkeit von der Prägung die Reaktion auf bestimmte Verhaltensweisen und Botschaften eines Anderen bestimmt. Beim „Versenden“ einer Nachricht spielen neben den Sach- und Beziehungs-Aspekten auch die Selbstoffenbarung und der Appell eine wichtige Rolle. Inwiefern all diese Komponenten das Verstehen und die Kommunikation beeinflussen, ist abhängig vom kulturellen Hintergrund und den Erfahrungen beider Gesprächspartner (Felsing, 2006, S. 3). Um das Verhalten des Gegenübers vorhersehen zu können, ist es notwendig, sich intensiv mit seiner Kultur zu befassen. Umso besser die Vorbereitung, desto präziser sind die Vorhersagen.

Besonders interessant für die spanischen Klienten war jedoch das zweite Modell (Watzlawick, Beavin und Jackson), welches den Fokus auf die zwischenmenschliche Beziehung der Gesprächspartner legt. Die Hauptaussage ist, dass jegliche Kommunikation in hohem Maße durch die Beziehung geprägt ist. Sowohl das Gesagte als auch das Empfundene sowie Gestik und Mimik der Gesprächsteilnehmer sind abhängig von den Gefühlen und Emotionen zueinander (Seidel, 2007, S. 73).

Die persönliche Beziehung ist für die Spanier elementarer Bestandteil jedes Geschäftsverhältnisses und meist bedeutender als Konditionen, Produkte oder finanzielle Situation des Gegenübers. Für die meisten ist es daher unvorstellbar, Geschäfte mit verhassten oder ihnen unsympathischen Personen abzuschließen. Hingegen wird in Deutschland deutlich mehr auf Inhalte und 
Bedingungen der Verhandlungen geachtet. Durch diesen kulturellen Gegensatz kommt es immer wieder zu Abbrüchen von Verhandlungen, da die Spanier feststellen, dass die Chemie nicht stimmt.

Es ist für beide Seiten wichtig zu verstehen, dass der Fokus des Gegenübers auf einem anderen Aspekt liegt. Da Deutschland eine inhaltsbezogene Kultur hat, entgehen ihnen oft Signale, die der Gegenüber auf emotionaler Ebene sendet (Brinker Dozier, Husted,McMahon, 1998, S. 113). Bereits vor der ersten Kommunikation muss also auf die oben beschriebenen Aspekte hingewiesen werden, da diese Einfluss auf Art und Weise der Kontaktaufnahme haben. Ein Beispiel: Während Deutsche am liebsten alles schriftlich zugesendet bekommen, bevorzugen Spanier bereits im frühen Stadium ein Kennenlernen bei einem persönlichen Treffen.

Diese Unterschiede sind den Kunden häufig unbekannt, und sie denken darüber zunächst nicht nach - sondern erst, wenn sich negative Erfahrungen einstellen. Manche sind sich der Unterschiede bewusst, sind aber unsicher, wie sie damit umgehen und sich selbst vorbereiten sollten. Praktische Hinweise bezüglich der Kulturen und Verhaltensweisen können Missverständnisse und erfolglose Gespräche vermeiden. Anfragen in Bezug auf interkulturelle Kommunikation gehen in fast allen Abteilungen regelmäßig ein, oft in Kombination mit der Bitte nach weiteren Informationen über den fremden Markt. Dieses ist zumeist ein Zeichen dafür, dass der Kunde ernsthaftes Interesse am Zustandekommen einer bilateralen Geschäftsbeziehung hat.

\section{Auswertung des Praktikums}

Bei meinem Praktikum handelte es sich um ein Pflichtpraktikum im Rahmen des B.A.-Studiengangs Europäisches Management. Um ein solches angemessen zu bewerten, werden daher im Folgenden zwei ausschlaggebende Aspekte analysiert. Dies sind zum einen die Betrachtungsweise mit Bezug auf das Studium und zum anderen die persönliche Reflexion meiner Erwartungen und Erfahrungen.

Viele der im Studium behandelten Themen und Theorien können im Berufsalltag in der AHK angewandt werden. Aber die zu bearbeitenden Aufgaben sind sehr vielfältig. Es ist nicht möglich, auf jede einzelne vollständig vorbereitet zu sein. Insbesondere im direkten Kundenkontakt merkte ich, dass mir praktische Erfahrung fehlte. Hierbei erleichterte zwar das theoretische Wissen über Kommunikation das Handeln, jedoch waren besonders bei eskalierenden Situationen diese Kenntnisse nicht ausreichend, um sicher eine Deeskalation herbeizuführen. Diese Ungewissheit wurde zudem verstärkt, wenn die Kommunikation auf Spanisch stattfand.

Um solche Situationen für zukünftige Praktikanten zu vereinfachen, könnte man Schulungen zum Thema Kundenumgang anbieten, am besten in verschiedenen Fremdsprachen. In diesen Schulungen ließe sich sowohl das nötige Vokabular als auch eine Routine bezüglich atypischer Situationen erlernen.

Ein Beispiel aus meinem Praktikum war ein aufgebrachter Vater, der sich bei der AHK über die Arbeitsbedingungen seiner Tochter in einem deutschen Unternehmen beschwerte. Er war temperamentvoll und wütend. Er beleidigte sowohl mich als auch die Kammer. Weder beruhigende noch strenge Worte konnten ihn bremsen. Ich war etwas schockiert, und mir fehlte das passende Vokabular. Es war mir unmöglich, für eine Deeskalation zu sorgen und die Situation für beide Seiten vorteilhaft zu lösen.

Obwohl unsere Hochschule im Vergleich wohl deutlich besser als andere auf den praktischen Teil der Arbeit vorbereitet, so hatte ich doch nie eine komplette Marktanalyse durchgeführt. Eine Herleitung der Analysestruktur ist zwar aufgrund der erlern- ten Theorie und praktischen Erfahrungen im Bereich Recherche und Datenaufbereitung möglich, sorgt jedoch anfänglich für Verunsicherung. Man könnte ein solches Projekt für ein selbst ausgewähltes Unternehmen bereits während der ersten vier Semester durchführen. Ebenso wäre es sinnvoll, Lehrveranstaltungen zum Internationalen Management, welche erst im fünften und sechsten Semester gelesen werden, vorziehen, da sich z.B. die AHK im Wesentlichen mit Exportvorhaben oder Markteinstieg in fremden Ländern beschäftigt, was exakt dem Lehrinhalt dieser Veranstaltung entspricht.

Bis auf die beiden angeführten Aspekte fühlte ich mich jedoch gut vorbereitet, da es mir möglich war, alle Aufgaben selbstständig zu lösen. Dies ist mit dem nötigen Hintergrundwissen und der Erfahrung im selbstständigen Erarbeiten von Themenfeldern zu begründen, wodurch meine anfängliche Unsicherheit sank.

Doch wäre es hilfreich gewesen, bereits während der ersten Semester stärker auf gewisse Unterschiede und auch kritische Missstände in europäischen Staaten hinzuweisen. Durch Gespräche in Spanien wurde mir zum ersten Mal bewusst, welche strukturellen Probleme das Land jenseits der aktuellen Wirtschaftskrise hat. Diese wird aus Sicht vieler Spanier insbesondere durch Korruption und familiäre Beziehungen oder auch Seilschaften begünstigt. Es wird vielen Politikern unterstellt, nicht aktiv genug gegen diese Probleme vorzugehen, da diese persönlich davon profitierten. In Spanien ist daher eine zunehmende Politikverdrossenheit festzustellen.

Genehmigungen für Geschäfte, hieß es, würden häufig durch Schmiergelder gefördert - oder durch „Vitamin B“. Damit ist aber nicht dasselbe gemeint wie in Deutschland. Vielmehr handelt es sich hierbei um familiäre Verbindungen. Dies geht soweit, dass in Madrider Universitäten, junge Studentinnen mit Heiratsaufträgen zu Vorlesungen geschickt wurden. Der Wunsch der Eltern war, dass die Tochter einen Partner findet, von dem sie wirtschaftlich profitieren könnten. Politikersöhne schienen sehr begehrt, ebenso wie Nachkommen ehemals wichtiger Adliger. Als ich mein Unverständnis äußerte, erklärte mir ein befreundeter Geschäftsmann, dass es nahezu unmöglich sei, sich nicht den Regeln dieses Systems zu unterwerfen, da dies zwangsläufig zum unternehmerischen Misserfolg führe. Durch Recherchen war ich mir zwar bewusst, dass das Thema Korruption, besonders im Bauwesen, eine erhebliche Rolle in der Wirtschaftskrise Spaniens spielte, nicht aber, dass es in so gut wie allen politischen und wirtschaftlichen Bereichen präsent ist. Eine systematische und kritische Auseinandersetzung mit europäischen Wirtschaftssystemen wäre hilfreich gewesen, um adäquat auf diese Situation reagieren und zudem die Situation in einem krisengeplagten Land besser nachvollziehen zu können.

Im Allgemeinen bereitet ein interdisziplinärer Wirtschaftsstudiengang, welcher zusätzlich Themengebiete wie Recht und Politik beinhaltet, jedoch gut auf Tätigkeiten in der AHK vor. Deutlich wurde mir die Notwendigkeit eines solchen Studiums im Gespräch mit den Mitarbeitern, welche mehrheitlich ein vergleichbares Studium absolviert hatten und im Anschluss häufig zuerst für IHKen in Deutschland tätig waren. Während dieser Zeit konnten sie sich bereits mit den Abläufen, Zielen und Funktionen der IHK-Organisation sowie ihrem wirtschaftspolitisch geprägten Umfeld vertraut machen. Dies ist gerade in der aktuellen wirtschaftlichen Situation Spaniens wichtig, da ein Verständnis von Zusammenhängen zwischen Politik und Wirtschaft erforderlich ist und auch verlangt wird. Möglichst jede Handlung der AHK sollte dazu dienen, die wirtschaftliche Situation Spaniens durch die Vermittlung oder Verbesserung von Geschäftsbeziehungen ins vergleichsweise krisensichere Deutschland zu verbessern.

In diesem Zusammenhang gewinnt die beratende Funktion der AHK immer mehr an Bedeutung, nicht etwa nur für die Unternehmen selbst, sondern auch für die Regierungen beider Staaten, 
da diese die politischen und rechtlichen Grundlagen schaffen müssen. In der Zukunft wird die Unterstützung spanischer - nicht nur deutscher - Unternehmen eine immer größere Rolle spielen. Diese Entwicklung ist bereits zu beobachten, da durch gezielte Marketingaktionen wie zum Beispiel Mailings, Publikationen, Anzeigen in Zeitungen und anderen Medienauftritten immer mehr spanische Kunden akquiriert werden können. Diese Initiative geht nicht etwa ausschließlich von der AHK selbst aus, um die Eigenfinanzierungsquote zu erhöhen, sondern auch von der deutschen Regierung, die sich dadurch eine wesentliche Verbesserung der spanischen Wirtschaftskraft und der Verflechtung der Märkte erhofft.

Zusammengefasst setzt das Arbeiten in einer Einrichtung wie der AHK ein wirtschaftliches und politisches Grundverständnis voraus, da die Aufgaben nur so optimal zu erfüllen sind. Unser Studiengang bildet durch die theoretische Vorbereitung, aber besonders die Praxisbezogenheit eine gute Basis für ein solches Praktikum. Es ist EM-Studenten daher möglich, schnell selbstständig in diesem Bereich tätig werden können. Festzustellen ist, dass bei einem solchen Praktikum das erlernte Wissen Anwendung findet und die Kenntnisse in vielen Bereichen vertieft oder sogar durch praktische Erfahrungen ergänzt werden. Ein Praktikum bei einer AHK trägt aufgrund des Aufgabenbereiches, aber auch wegen der Stellung der Institution zum Studienerfolg bei.

\section{Reflexion persönlicher Erwartungen}

Unabhängig von der hochschulischen Sicht aufs Praktikum spielen auch persönliche Erwartungen und der Nutzen des Praktikums für das eigene Leben eine wesentliche Rolle bei der abschließenden Bewertung. Meine Erwartung war, dass sowohl meine beruflichen Erfahrungen als auch mein Wissen aus dem Studium erweitert und gefestigt werden. Dies war der Grund, warum ich mich für ein Praktikum entschied, was bisherige berufliche Erfahrungen im politischen Bereich mit wirtschaftlichen Aspekten des Studiums verbindet. Hierfür kamen in meinem Fall genau zwei Optionen in Frage: ein Praktikum bei der Europäischen Union oder der Auslandshandelskammer. Beide Stellen hätten die von mir selbst vorgegebenen Voraussetzungen erfüllt, jedoch mit unterschiedlicher Gewichtung. Während etwa bei einem EU-Abgeordneten der Fokus auf Politik mit Schwerpunkt Wirtschaft gelegen hätte, handelte es sich bei der Stelle in der AHK um ein wirtschaftlich orientiertes Praktikum, welches lediglich ein erweitertes politisches Allgemeinwissen voraussetzte.

Die Entscheidung fiel mir sehr schwer. Ich entschied mich jedoch für die AHK, da ich im politischen Bereich dank einer Anstellung im Bundestag und meiner Parteiarbeit bereits mehr Erfahrung sammeln durfte. $\mathrm{Zu}$ meinem Bedauern musste ich feststellen, dass die Tätigkeit in Madrid fast ausschließlich Marketingbereiche umfasste und lediglich der administrative Teil einen politischen Aspekt beinhaltete. Dadurch war es mir möglich, mein politisches Wissen bezüglich beider Staaten immerhin bei der Kundenberatung anzuwenden. Allerdings hatte ich mir aufgrund der Stellenbeschreibung mehr Tätigkeiten in diesem Bereich erhofft. Glücklicherweise konnte ich jedoch meine Aufgaben sehr frei gestalten und somit bei einigen weiteren Themen politische Aspekte mit einbringen, zum Beispiel bei Marktanalysen. In Bezug auf meine beruflichen Tätigkeiten konnte ich zwar wenig inhaltlich arbeiten, jedoch den Bereich der Kommunikation mit Kunden bzw. Interessenten verbessern.

Ich konnte zwar bei meinen Aufgaben theoretisches Wissen anwenden, ich habe mir jedoch relativ wenig neues Wissen aneignen können. Besonders interessant war diese Erfahrung im Bereich Marketing, da mir zum ersten Mal bewusst wurde, wie theoretische Aspekte der Vorlesungen für Unternehmen bei der Planung relevant sind und welche Faktoren ihr Handeln zusätz- lich beeinflussen. Die AHK ist ein international agierendes Unternehmen, was mir die Möglichkeit gab zu verstehen, welche Faktoren allein aufgrund der Internationalität berücksichtigt werden müssen. Für die immer wachsende Anzahl von Unternehmen, die bisher ausschließlich national agiert hatten, war es besonders kompliziert zu überblicken, welche neuen Einflüsse beachtet werden müssen. In diesem Zusammenhang wurde mir abermals der Vorteil unseres Studiums gegenüber einem herkömmlichen BWL-Studiengang bewusst.

Bei der Bewerbung war ich sehr nervös, da die AHK besonders bezüglich der Sprache hohe Erwartung an die Kandidaten hat. Die meisten Stellen werden zumeist auf den Internetseiten von Universitäten oder auf Portalen wie „Madrid für Deutsche“ (madridfuerdeutsche.com) ausgeschrieben. Allerdings werden auch Initiativbewerbungen berücksichtigt. Auf den OnlinePlattformen wird meistens sowohl ein „Europass“ als auch alle Zeugnisse und ein persönliches Anschreiben erwartet. All dies muss sowohl in Deutsch als auch in Spanisch eingereicht werden, damit bereits im Vorfeld die Sprachkenntnisse der Kandidaten überprüft werden können.

In der zweiten Bewerbungsphase wird ein Telefoninterview durchgeführt, bei welchem neben Fachwissen abermals die Sprachkenntnisse und die Kommunikationsfähigkeit der Bewerber getestet werden. Bereits kurze Zeit später erhält der Kandidat dann per E-Mail eine $\mathrm{Zu}$ - oder Absage. In meinem Fall handelte es sich um eine Initiativbewerbung um die Stelle im Bereich Markt- und Absatzberatung. Der restliche Bewerbungsprozess war standardisiert; die Normen werden jedoch von Abteilung zu Abteilung unterschiedlich strikt befolgt. Es stellte sich heraus, dass ein Interview lediglich in einigen Bereichen durchgeführt wird, während bei anderen der Lebenslauf und das Anschreiben als alleinige Entscheidungsgrundlage dienen.

Aufgrund des streng regulierten Bewerbungsprozesses befürchtete ich, dass auch der gesamte Arbeitsalltag überreguliert sein würde. Dies war aber nicht der Fall. Obwohl ein grober Tagesablauf vorgegeben war, lie $\beta$ mein Chef mir viele Freiheiten. Die Reihenfolge der Aufgabe legte ich zumeist selbst fest und musste ihm lediglich bereits beim morgendlichen Teammeeting mitteilen, wie viel Zeit ich für jede Tätigkeit benötigen werde. Außerdem gehörten regelmäßige Zwischenberichte zum Ablauf eines jeden Tages.

Die oben beschriebenen Aufgaben fielen mir mit jedem Tag leichter, da es sich beim Großteil um Routineaufgaben handelte. Dennoch kamen jeden Tag neue Kunden mit komplett neuen Ansprüchen hinzu, die vorab mit meinem Chef besprochen werden mussten. Gerade am Anfang nahmen sich sowohl mein Vorgesetzter als auch meine Kollegin sehr viel Zeit, um mir möglichst detailliert die Abläufe zu erklären.

Insgesamt war die Aufnahme sehr herzlich und freundlich und erleichterte mir in hohem Maße die Integration in das Team. Durch die angenehme Atmosphäre und das freundliche Miteinander fiel mir der Abschied sehr schwer, zumal gerade im Sommer in Spanien mehr Zeit für das Zwischenmenschliche bleibt. Dieses liegt zum einen daran, dass die Arbeitszeit verkürzt wird und dadurch die Pausen entfallen, und zum anderen daran, dass sich ab Mitte Juli die Anzahl der Anfragen und Aufträge reduziert. Der Vorteil ist, dass sowohl alte Aufgaben aufgearbeitet werden können, aber auch die Kontakte zu Kollegen intensiviert werden.

Persönlich hat mir das Praktikum sehr viel gebracht, sowohl die positiven Aspekte als auch die negativen. Es war für mich sehr erschreckend, wie schlecht Unternehmen oft informiert sind und wie viele Chancen sie sich durch eine übertrieben konservative Unternehmensphilosophie entgehen lassen. Die Investitionsfreudigkeit in Spanien ließ bei vielen Unternehmen zu wünschen übrig, da sie Angst vor Fehlinvestitionen haben. Dieses Handeln brachte die Mitarbeiter regelmäßig zur Verzweiflung, da die 
guten Ratschläge und Analysen oft nicht angenommen und umgesetzt wurden. Trotz des Verständnisses, welches ein Jeder für die spanischen Unternehmen hatte, fiel es oft schwer, die Entscheidungen zu akzeptieren und nicht weiter zu versuchen, den Kunden von der für uns besser erscheinenden Alternative zu überzeugen.

\section{Fazit}

Insgesamt war das Praktikum in der Deutschen Handelskammer für Spanien eine absolut positive Erfahrung, da sie eine Bereicherung für verschiedene Bereiche des Lebens war. Negative Erfahrungen gehörten dazu, aber sie entpuppten sich meist eher als Herausforderungen. Dass ich Aufgaben bereits nach kürzester Zeit selbstständig lösen konnte, bestärkte das Gefühl, ein vollständiges Teammitglied zu sein. Ein Land wie Spanien, welches aktuell viele Veränderungen im wirtschaftlichen Bereich durchlebt, ist besonders interessant für die Arbeit einer $\mathrm{AHK}$. Das Gefühl, nicht mehr objektive Beobachterin, sondern plötzlich Vermittlerin zwischen zwei Wirtschaftsmächten zu sein, fordert und befördert ein verändertes und größeres Denken. Hierfür ist es unbedingt nötig aufzuhören, ,typisch deutsch“ zu denken. Eine solche Arbeit ist stets mit neuen politischen und wirtschaftlichen Herausforderungen verbunden und war somit für mich interessant und lehrreich.

\section{Links}

$\rightarrow$ Deutsche Handelskammer für Spanien / Cámara de Comercio Alemana para España: www.ahk.es

$\rightarrow$ Netzwerk der Deutschen Auslandshandelskammern (AHK) mit 120 Standorten in 80 Ländern auf 5 Kontinenten: www.ahk.de

$\rightarrow$ Deutscher Industrie- und Handelskammertag (DIHK): www.dihk.de

$\rightarrow$ Germany Trade \& Invest: www.gtai.de

$\rightarrow$ Deutsches Außenwirtschaftsportal iXPOS: www.ixpos.de

$\rightarrow$ Portal „Madrid für Deutsche“ www.madridfuerdeutsche.com 


\section{Quellen}

Brinker Dozier, Husted, McMahon (1998): Teaching Business Ethics. Need for approval in low-context and high-context cultures: a communications approach to cross-cultural ethics. Kluwer Academic Publishers, S. 113.

Deutsche Bundesbank (2012): Leistungsbilanzdefizite in den Peripherieländern rückläufig., Deutsche Bundesbank, S. 22. Online verfügbar unter

http://www.bundesbank.de/Redaktion/DE/Downloads/Veroeffentlichu ngen/Monatsberichtsaufsaetze/2012/2012_11_umfeld.pdf?_blob=pub licationFile.

Deutsche Handelskammer für Spanien (2013): Intranet: AHK. Informationen über die Aufbau, Strukturen und Funktionen der AHK Spanien, bestehend aus 20 Dokumenten, zuletzt geprüft am 30.08.2013.

Deutsche Handelskammer für Spanien (2013): Jahrbuch 2012/2013. Hg. V. Deutsche Handelskammer für Spanien. Madrid.

Dow Jones (2013): Spanien muss höheres Defizit für 2012 einräumen. In: Frankfurter Allgemeine Zeitung 2013, 27.03.2013, S.1. Online verfügbar unter

http://www.faz.net/aktuell/wirtschaft/eurokrise/spanien/schuldenkrisespanien-muss-hoeheres-defizit-fuer-2012-einraeumen-12130044.html.

Eurostat: Arbeitsproduktivität je Beschäftigten Index (EU27 = 100). Online verfügbar unter

http://epp.eurostat.ec.europa.eu/tgm/table.do?tab=table\&init=1\&plugi $\mathrm{n}=1$ \&language $=$ de\&pcode $=$ tec00116, zuletzt geprüft am 29.09.2013.

Eurostat (2013): Nominale Lohnstückkosten (2005 = 100) - jährliche Daten. Online verfügbar unter http://epp.eurostat.ec.europa.eu/tgm/table.do?tab=table\&init=1\&plugi $\mathrm{n}=1$ \&language $=$ de \&pcode $=$ tipslm20, zuletzt geprüft am 29.09.2013

Felsing, Tobias (2006): Kommunikationsprobleme in Teams mit hoher aufgabenbezogener Diversität: Ursachen und Möglichkeiten zur Intervention: VS Verlag für Sozialwissenschaften / Springer Fachmedien Wiesbaden GmbH Wiesbaden (Gruppendynamik und Organisationsberatung), S. 3 .

Freiling, Jörg; Reckenfelderbäumer, Martin (2007): Markt und Unternehmung. Eine marktorientierte Einführung in die Betriebswirtschaftslehre. 2. Aufl. Wiesbaden: Gabler, S. 199-202. Online verfügbar unter http://dx.doi.org/10.1007/978-3-8349-9536-0.

García-Berdoy, Pablo (2013): Deutsch-Spanische Beziehungen: Bilanz des Jahres 2012. In: Deutsche Handelskammer für Spanien (Hg.): Jahrbuch 2012/2013. Madrid, S. 22-24.

Georg Oster (2013): Spaniens notwendige Neupositionierung. Germany Trade and Invest. Deutsche Handelskammer für Spanien. Madrid, 05.08.2013.

Griese, Kai-Michael; Bröring, Stefanie (2011): Marketing-Grundlagen. Eine fallstudienbasierte Einführung. 1. Aufl. Wiesbaden: Gabler Verlag / Springer Fachmedien Wiesbaden GmbH Wiesbaden, S. 71-83. Online verfügbar unter http://dx.doi.org/10.1007/978-3-8349-6622-3.

Hiller, Kerstin (2007): Werbung als Schlüsselfaktor bei der Einführung neuer Produkte. 1. Aufl. s.l: DUV Deutscher Universitäts-Verlag, S.21. Online verfügbar unter http://gbv.eblib.com/patron/FullRecord.aspx?p=749201.

Hudetz, Kai; Kaapke, Andreas (2009): Lexikon Handelsmanagement. Controlling - Führung - Marketing. 1. Aufl. s.l: Verlagsgruppe Deutscher Fachverlag (Enzyklopädie des Handels), S. 223. Online verfügbar unter http://ebooks.ciando.com/book/index.cfm/bok id/21202.

Kemper, Hans-Georg; Baars, Henning; Mehanna, Walid (2010): Business Intelligence - Grundlagen und praktische Anwendungen. Eine Einführung in die IT-basierte Managementunterstützung. 3. Aufl. Wiesbaden: Vieweg+Teubner Verlag / GWV Fachverlage GmbH Wiesbaden (Studium), S. 154. Online verfügbar unter http://dx.doi.org/10.1007/978-3-8348-9727-5.

Kuß, Alfred; Tomczak, Torsten; Reinecke, Sven (2008): Marketingplanung. Einführung in die marktorientierte Unternehmens- und Geschäftsfeldplanung. 5. Aufl. s.1: Gabler Verlag, S. 222. Online verfügbar unter http://ebooks.ciando.com/book/index.cfm/bok_id/331430.

Meffert, Heribert; Burmann, Christoph; Kirchgeorg, Manfred (2012): Marketing. Grundlagen marktorientierter Unternehmensführung; Konzepte - Instrumente - Praxisbeispiele. 11. Aufl. Wiesbaden: Gabler Verlag (Meffert-Marketing-Edition), S. 22. Online verfügbar unter http://dx.doi.org/10.1007/978-3-8349-6916-3.
Müller, Ragnar; Plieninger, Jürgen; Rapp, Christian (2013): Recherche 2.0. Finden und Weiterverarbeiten in Studium und Beruf: Springer VS, S. 17-21.

Plettenberg, Walther (2013): Geschäftsleitung. In: Deutsche Handelskammer für Spanien (Hg.): Jahrbuch 2012/2013. Madrid, S. 48-51.

Pufahl, Mario (2006): Vertriebscontrolling. Wiesbaden: Springer Fachmedien, S. 136. Online verfügbar unter http://gbv.eblib.com/patron/FullRecord.aspx?p=750803.

Röhl, Thomas (2013): Fachkräftemangel hausgemacht. In: Packreport 2013, 13.09.2013 (09), S. 3. Online verfügbar unter http://www.wisonet.de/webcgi?ST ART $=$ A60\&DOKV_DB $=$ ZGEN\&DOKV_NO=PAC $\mathrm{K} 2013091283298 \& D O K V \_H S=0 \& \mathrm{PP}=1$.

Seidel, Alina (2007): Kundenorientierte Kommunikation. 1. Aufl. s.l: DUV Deutscher Universitäts-Verlag, S. 73. Online verfügbar unter http://gbv.eblib.com/patron/FullRecord.aspx?p=751489.

Senden, Manfred J.; Dworschak, Johannes (2012): Erfolg mit Prozessmanagement. Nicht warten bis die "Gurus" kommen. 1. Aufl. Freiburg: Haufe (Haufe Sachbuch Wirtschaft - Band 01311), S. 47-61. Online verfügbar unter http://sub-hh.ciando.com/book/?bok_id=291593.

Servicio Público de Empleo Estatal (2013): Formación profesional dual Contrato para la formación y el aprendizaje. Hg. v. Ministerio de empleo y seguridad social. Madrid, S.1. Online verfügbar unter http://www.sepe.es/contenido/conocenos/publicaciones/pdf/FPD Cont rato_FyA.pdf.

Simon, Walter (2009): Managementkonzepte von A bis Z. 1. Aufl. s.1: Gabal Verlag GmbH, S. 146-151. Online verfügbar unter http://ebooks.ciando.com/book/index.cfm/bok_id/36325.

Söllner, Fritz; Wilfert, Arno (2001): Die Zukunft des Sozial- und Steuerstaates. Festschrift zum 65. Geburtstag von Dieter Fricke. Heidelberg: Physica-Verlag HD, S. 470-488. Online verfügbar unter http://dx.doi.org/10.1007/978-3-642-57606-5.

Statista: Spanien: Arbeitslosenquote von 2003-2013. 2013. Online verfügbar unter http://de.statista.com/statistik/daten/studie/17327/umfrage/arbeitslosen quote-in-spanien/, zuletzt geprüft am 28.09.2013.

Tropp, Jörg (2011): Moderne Marketing-Kommunikation. System Prozess - Management. 1. Aufl. Wiesbaden: VS Verlag für Sozialwissenschaften / Springer Fachmedien Wiesbaden GmbH Wiesbaden, S.59. Online verfügbar unter http://dx.doi.org/10.1007/978-3-53192802-9. 